\title{
The Effect of Synthetic Vision Enhancements on Landing Flare Performance
}

\author{
L. Le Ngoc, ${ }^{*}$ C. Borst,$^{\dagger}$ M. Mulder, ${ }^{\ddagger}$ and M. M. van Paassen ${ }^{\S}$ \\ Delft University of Technology, Faculty of Aerospace Engineering, Delft, The Netherlands
}

\begin{abstract}
The usage of heads-down, non-conformal synthetic vision displays for landings below minimums has inherent problems during the flare due to minification effects. Literature showed that pilots can use four visual cues to perform a manual flare maneuver. Amongst their strategies, the Jacobson flare method seemed the most suitable to provide flare initiation and flare control cues. Offline, linear simulation results indicated that the Jacobson method was robust and its performance was comparable to an automatic landing system. An enhanced synthetic vision display was designed offering support cues to aid pilots with this landing method. An experiment was conducted in a fixed-based simulator with eight professional pilots and eight novice pilots. The enhanced display was tested against a basic version and conventional primary flight display with outside visuals. Each pilot group was split into two groups with different display orders to check for possible learning effects. The experiment showed that touchdown position was not affected by display type. The enhanced display did achieve softer touchdown sink rates compared to the basic version. The softest landings were done on outside visuals. A strong learning curve was also observed for the group of pilots that first flew with the enhanced display: this translated into better achieved performances with subsequent displays. Therefore, it could be a valuable tool for basic flight training and reduce the number of flight hours required in mastering the landing flare.
\end{abstract}

\section{Introduction}

CURREnT regulations forbid aircraft to fly Visual Flight Rules (VFR) under Instrument Meteorological $\cup$ Conditions (IMC). ${ }^{1}$ That is, flight operations into non-precision equipped airports in bad weather or night time are not possible. The emergence of Synthetic Vision System (SVS) and Enhanced Vision System (EVS) displays have been identified as technology enablers to address these limitations. ${ }^{2}$ These modern displays provide pilots with synthetic, infrared or radar image cues when outside visibility is severely reduced. This concept of flying is referred to as Electronic Visual Flight Rules (EVFR). Both SVS, EVS and hybrid displays are already commercially available for high-end business jets and have proven to extend the operational flexibility of their operators. ${ }^{3}$ However, the usage of SVS for landings below minimums is not allowed: pilots are required to transition to outside visuals prior to touchdown. ${ }^{2}$ SVS usage without cross-checking with a penetrating sensor like EVS can lead to unsafe conditions e.g. runway incursion or runway database misalignment. ${ }^{4}$ Perspective distortions of being a heads-down, non-conformal display and minification makes it hard for pilots to judge distances, altitudes and time-to-contact during the final seconds of landing. ${ }^{5}$ Solving these problems during landing can contribute towards the acceptance of true EVFR operations in the future.

${ }^{*}$ MSc student, Control and Simulation Division, Faculty of Aerospace Engineering, Delft University of Technology. Kluyverweg 1, 2629 HS Delft, The Netherlands. E-mail: luanlengoc@hotmail.com

${ }^{\dagger} \mathrm{PhD}$, Control and Simulation Division, Faculty of Aerospace Engineering, Delft University of Technology. Kluyverweg 1, 2629 HS Delft, The Netherlands. E-mail: c.borst@tudelft.nl

${ }^{\ddagger}$ Professor, Control and Simulation Division, Faculty of Aerospace Engineering, Delft University of Technology. Kluyverweg 1, 2629 HS Delft, The Netherlands. E-mail: m.mulder@tudelft.nl

$\S$ Associate Professor, Control and Simulation Division, Faculty of Aerospace Engineering, Delft University of Technology. Kluyverweg 1, 2629 HS Delft, The Netherlands. E-mail: m.m.vanpaassen@tudelft.nl 
This paper aims at studying the longitudinal aspects of landing flare techniques and the visual cues that are available in the environment to determine how to best utilize this information in a pilot support display to enable consistent touchdowns in zero-zero visibility conditions. The research comprises two parts: (1) a theoretical part analyzing various landing flare techniques, and (2) an experiment evaluation validating the theoretical findings.

\section{Theoretical analysis}

\section{A. Automatic flare control}

Much about the flare maneuver can be learned from analyzing an autoland control system. The basic operation of automatic flare control is the execution of a pre-set pitch command profile by the autopilot as a function of vertical speed. Advanced autoland systems are also able to control thrust (reduction and/or reversal), wheel- and airbrakes deployment as well as ground steering upon touchdown. A classic flare control law was derived from flight test data, ${ }^{6}$ which revealed that pilots tend to arrest their vertical sink rate in an exponential manner during the flare. The autoland system can be programmed with this characteristic exponential path, mathematically described by:

$$
h=h_{0} e^{-t / \tau}
$$

where $h$ is the altitude above the runway, $h_{0}$ the altitude upon flare initiation, $t$ the elapsed time since flare start and $\tau$ the exponential path constant. The value for $\tau$ depends on the geometrical path and is influenced by the desired ground distance traveled until touchdown. Differentiating (1) reveals the vertical speed which is the control variable for the flare:

$$
\dot{h}=-\frac{h_{0}}{\tau} e^{-t / \tau}=-\frac{h}{\tau}
$$

\section{B. Manual flare}

Pilots perform the manual flare in a similar way as the autopilot described previously. However, the reference signal to perform the flare is too complex for humans to execute. Even if the required pitch was available on a command display, human lag would make it impossible to follow. ${ }^{7}$ Pilots rely on traditional visual cues in order to time the flare initiation and execution. The success of the flare depends on pilot skill, judgment and experience.

\section{Flare initiation}

There are three possible visual cues identified in literature to time the flare:

- Critical perceived distance

- Critical perceived runway angle

- Critical perceived time-to-contact

CRITICAL PERCEIVED Distance This strategy is similar to an automatic flare and can be found in flight instruction manuals: dependent on aircraft type, pilots start the flare when they perceive the aircraft reaching an appropriate height above the runway. Instead of an appropriate height, an absolute distance from a desired aimpoint or the end of the runway can also be used. 
CRItical Perceived Runway Angle The flare is initiated when the visual angle $(\psi)$, posed by the left and right edges of the runway at at a certain tangent line, reaches a critical value. ${ }^{8}$ This is illustrated in Figure 1. This strategy is not universally applicable: if a pilot adopts this value for a known runway and executes it on an unfamiliar one, it could lead to serious errors. A critical angle $\psi$ on a narrow runway would result in an early flare when attempting the same on a wider runway. Since the geometry would differ for a different glideslope, the critical angle $\psi$ is only valid for a particular glideslope and its appropriate flare height.

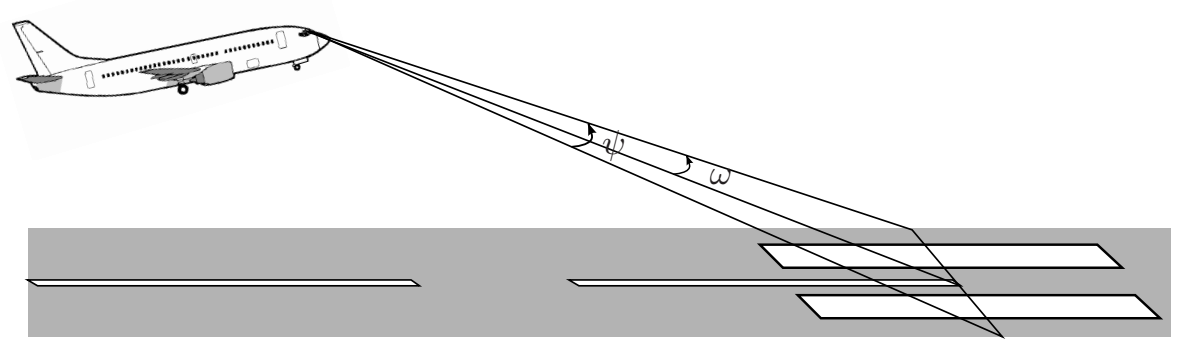

Figure 1. Two visual angles for flare timing $(\psi$ and $\omega)$

CRITICAL PERCEIVED TIME-TO-CONTACT Critical perceived time-to-contact (TTC) is a strategy for pilots to start the flare when their perceived TTC with the runway reaches a critical value. ${ }^{9,10}$ This strategy assumes a pilot can perceive the TTC accurately in an unbiased manner when the aircraft's main gear will contact the runway with no action taken. ${ }^{8}$ Mathematically, TTC is defined as the distance between the pilot's eye and the aiming point divided by the closing velocity. Calculation in this manner requires both accurate depth and velocity perception. However, the human visual system is not capable of this.

Advantage can be taken of the angular relation that provides the TTC without the need to directly estimate speed and distance:

$$
T T C=\frac{x}{\dot{x}}=\frac{\sin \omega}{\omega} \approx \frac{\omega}{\dot{\omega}},
$$

where $\omega$ is the visual angle between the centerline aimpoint and another point on the same aiming line and $\dot{\omega}$ the angular rate of change over time.

This offers a good strategy as this visual angle can be sensed more accurately from both the outside visual view as well as on a SVS display. TTC estimation should be improved when ground texture is available thereby allowing redundant estimates of TTC. ${ }^{8}$ As a result during experiments, when only the runway outline was visible, pilots used the perceived runway angle strategy and when the additional ground textures became sufficiently visible to get a good TTC estimation, switched to the TTC strategy. ${ }^{8}$ A drawback of using TTC is that it requires pilots to focus on the aimpoint to get their TTC estimate ${ }^{8}$ but this opposes flight instruction that brief pilots to move their viewing point away from the aimpoint to the far end of the runway just prior to starting the flare. ${ }^{11}$ Theoretical investigation of this strategy ${ }^{9}$ has also lead to an experimental command display to assist pilots in performing the flare. ${ }^{12}$ But the control variable used in that display could not be taken as a good measure to predict or control sink rates upon touchdown. Furthermore, the algorithm did not correct for any deviations in the approach prior to the flare. Flying the command display cues led to higher touchdown sink rates when the aircraft was high during the approach and having a high initial vertical speed.

\section{Flare execution}

Literature primarily covers control strategies for flare initiation. ${ }^{11}$ Research is sparse regarding flare execution. ${ }^{10}$ Flare execution appears to be relegated to anecdotal instructions in basic training manuals and more of an acquired skill to be accumulated with every landing made. ${ }^{13}$ Flare execution can compensate for 
slight errors in flare initiation: a flare started too late can be corrected by a more aggressive pull-back on the stick. The use of TTC to close the gap between the current state and end state of the flare as a method is supported by some evidence in research. ${ }^{14}$ However, there is still much research to be done to provide a better understanding of the last phase of the flare.

\section{Landing flare with Synthetic Vision}

Inherent minification effects with SVS makes hard to judge distances. ${ }^{5}$ This means SVS without enhancemenst is not expected to be capable of providing adequate visual cues for the critical perceived distance strategy. The perceived runway angle strategy would work well with an SVS display since displaying angular relations is an inherent advantage of a perspective display. However, one still has the same problem having to cope with varying runway widths during operations. Coupled with minification effects, this makes this visual cue not a practical method. All of the aforementioned flare timing strategies also have constraints and limitations for operational use. This has been confirmed in a flight simulator study, where the pilots did not initiate their flares at either a constant height above the runway, or at some constant time before touchdown. ${ }^{15}$ Since the pilots were aiming to touchdown at a specific point on the runway instead of trying to only achieve a reduction in sink rate on touchdown to safe levels, their control strategy was not governed to a particular theoretical method.

\section{Jacobson flare}

The Jacobson flare ${ }^{13}$ offers a way for quantifying when to start the flare in combination with instruction on how to control the flare till touchdown. This method provides timing adjustments for the aircraft coming in either too steep or too shallow on the glideslope and compensation for different runway slopes. Runway slope compensation is not the purpose of this research, however. The principle of the Jacobson flare is based on simple triangulation that provides a visual fix to time the flare initiation. The flare point is derived from a pre-set longitudinal position on the runway centerline and later superimposed by the cockpit lower visual cut-off angle. As the aircraft descends on the glideslope, the cockpit cut-off angle will block the ground from the pilot's view underneath the nose. When the aircraft arrives at the correct flare height, a specific cut-off point on the runway will correspond to this geometry. If this cut-off point is learned beforehand, the pilot will have a means to estimate, monitor and trigger the flare accurately. The Jacobson flare method originally uses small angles approximation and simple estimated values so pilots can perform the calculations quickly.

\section{A. Approach geometry}

The approach geometry for the Jacobson flare is illustrated in Figure 2. The distance between the aimpoint back to the impact point, $\Delta x$, is the sum of two geometrical components:

$$
\Delta x=x_{1}+x_{2}
$$

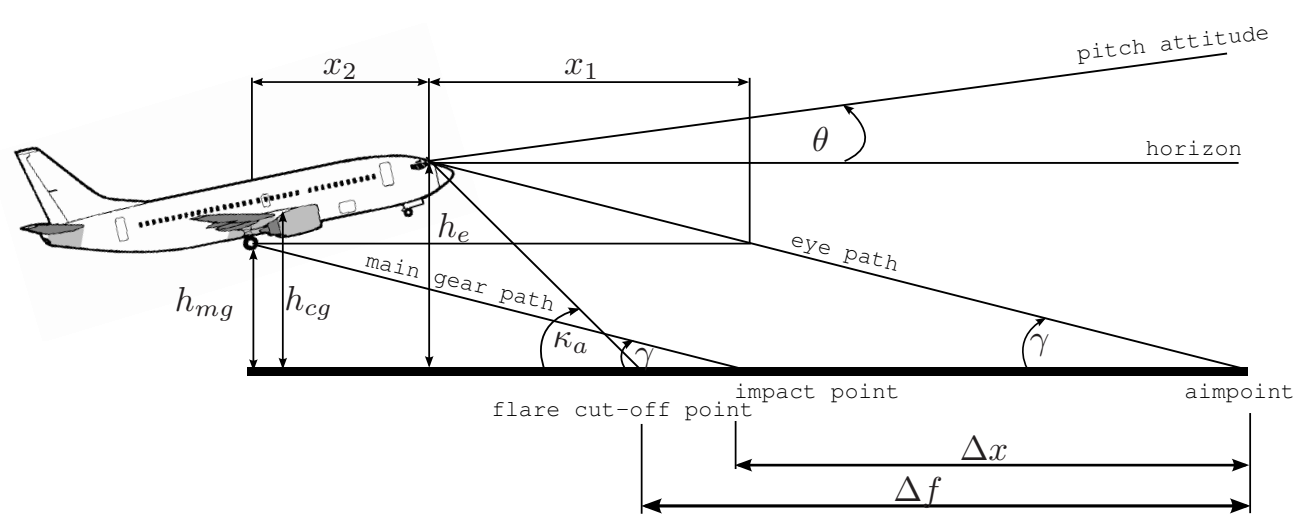

Figure 2. Jacobson flare geometry 
The geometrical component $x_{1}$ is obtained from the following relation:

$$
x_{1}=\frac{h_{e}-h_{m g}}{\tan |\gamma|}
$$

Combining (4) and (5) yields:

$$
\Delta x=\frac{h_{e}-h_{m g}}{\tan |\gamma|}+x_{2}
$$

The aircraft's Instrument Landing System (ILS) antenna and not the aircraft's center of gravity tracks the glideslope beam. Therefore, horizontal distances of the center of gravity $d_{c g}$, main gear $d_{m g}$ and pilot's eye position $d_{e}$ relative to the ILS antenna $d_{I L S}$ need to be determined. The geometry for an approaching aircraft on glideslope when the ILS antenna is directly over the runway threshold is drawn in Figure 3.

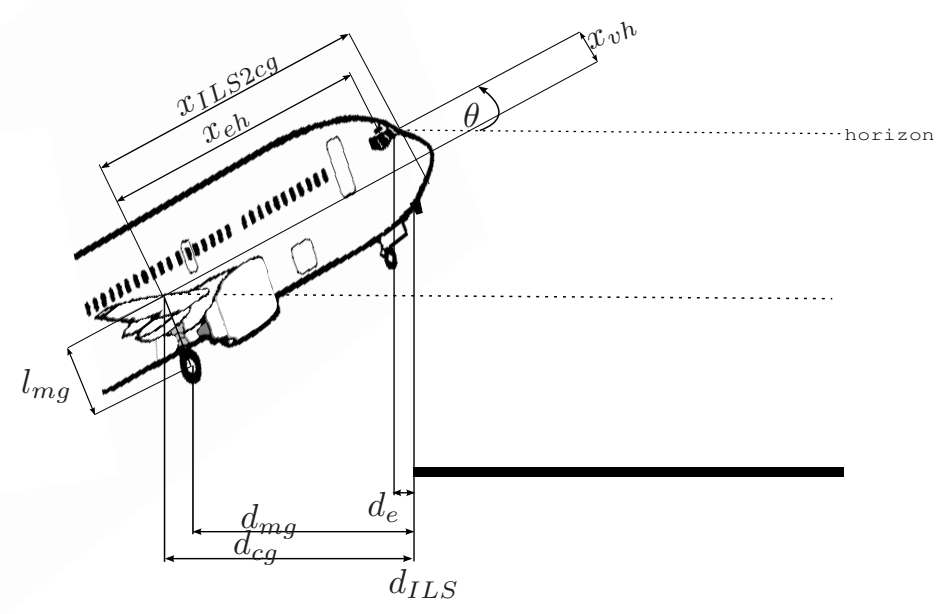

Figure 3. Aircraft geometry with ILS receiver antenna over runway threshold

The aircraft's center of gravity horizontal distance to the ILS antenna is approximated as:

$$
d_{c g}=d_{I L S}-x_{e h} \cdot \cos \theta
$$

It is assumed that the ILS antenna is located below the pilot's position and on the same fuselage level with the center of gravity. In this case $d_{I L S}=0$ per definition with the ILS antenna directly above the runway threshold and $x_{e h}$ is the distance between center of gravity and eye position.

The pilot eye position relative to the ILS antenna depends only on the vertical distance between the ILS antenna and eye $x_{v h}$ :

$$
d_{e}=d_{I L S}-x_{v h} \cdot \sin \theta
$$

The main gear strut length $l_{m g}$ is assumed a constant. The relative horizontal distance of the main gear to the ILS antenna is calculated by:

$$
d_{m g}=d_{c g}+l_{m g} \cdot \sin \theta
$$

The second geometrical component $x_{2}$ in (4) can be obtained from Figure 3 :

$$
x_{2}=d_{m g}-d_{e}
$$

Combining (7),(8) and (9) and substituting into (10) yields:

$$
x_{2}=\left(l_{m g}+x_{v h}\right) \cdot \sin \theta-x_{e h} \cdot \cos \theta
$$


As $x_{1}$ in (6) depends on $h_{e}$ and $h_{m g}$, these values can again be obtained from geometry. Another sketch of this situation and the geometry in Figure 4 shows the relative vertical distances.

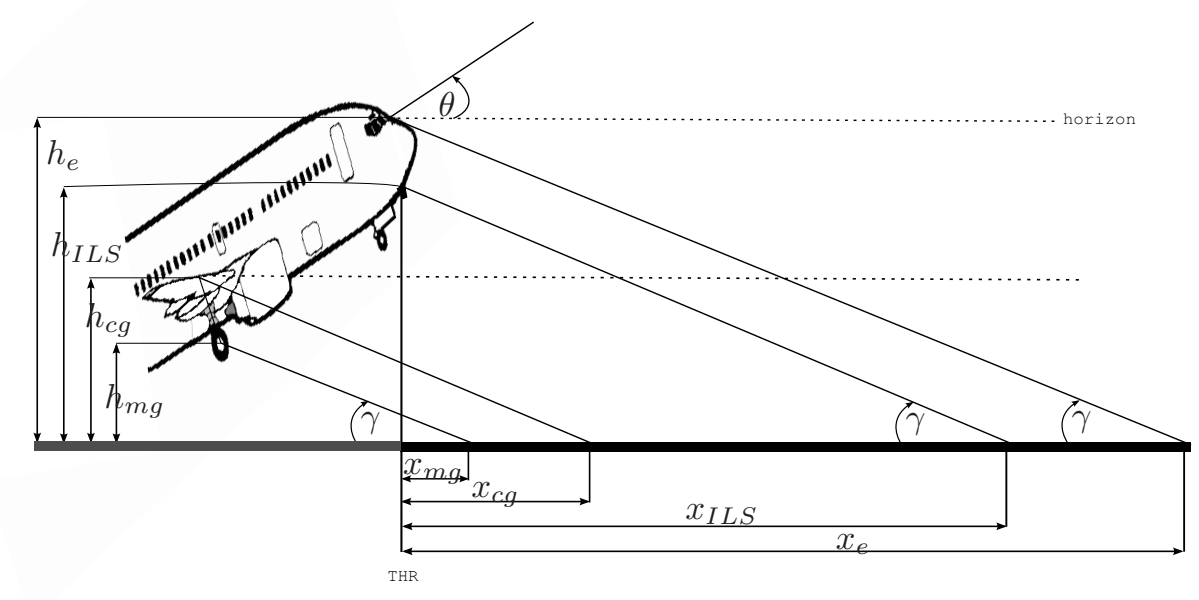

Figure 4. Approach aimpoint geometry with ILS receiver antenna over runway threshold

With the aircraft's ILS antenna tracking the glideslope and at a horizontal distance $x_{I L S}$ from the glideslope emitter, the altitude of the antenna $h_{I L S}$ is:

$$
h_{I L S}=x_{I L S} \cdot \tan |\gamma|
$$

Then the altitude of the center of gravity $h_{c g}$ is:

$$
h_{c g}=h_{I L S}-x_{e h} \cdot \sin \theta
$$

The altitude of the main gear is:

$$
h_{m g}=h_{c g}-l_{m g} \cdot \cos \theta
$$

And the altitude of the pilot's eye position is obtained by:

$$
h_{e}=h_{I L S}+x_{v h} \cdot \cos \theta
$$

Finally, combining (12), (13), (14), (15) and filling in (6) results in:

$$
\Delta x=\frac{\left(x_{v h}+l_{m g}\right) \cdot \cos \theta+x_{e h} \cdot \sin \theta}{\tan |\gamma|}+\left(l_{m g}+x_{v h}\right) \cdot \sin \theta-x_{e h} \cdot \cos \theta
$$

which can be rewritten per cosine and sine elements as:

$$
\Delta x=\left(\frac{x_{v h}+l_{m g}}{\tan |\gamma|}-x_{e h}\right) \cdot \cos \theta+\left(l_{m g}+x_{v h}+\frac{x_{e h}}{\tan |\gamma|}\right) \cdot \sin \theta
$$

Based on these equations, the location of the aimpoint and impact point on the runway for an approaching aircraft established on a particular glideslope can be determined. These equations also scale for various aircraft sizes and their relative component dimensions. 


\section{B. Flare initiation}

When the aircraft is on a stable, constant speed approach on glideslope and the aimpoint is constant with respect to the glareshield, it will reach the correct altitude to flare. The aimpoint and flare cut-off cues as seen from the cockpit are shown in Figure 5(a). At this point the pilot's eye position is defined as the flare eye altitude $h_{e f}$, which was determined from geometry. The cockpit lower cut-off angle $\kappa$ will ensure that the ground underneath the aircraft nose is blocked from view. At this flare initiation moment, there is a cut-off point on the ground with a distance $\Delta f$ from the aimpoint:

$$
\Delta f=\frac{h_{e f}}{\tan |\gamma|}-\frac{h_{e f}}{\tan \left|\kappa_{a}\right|}
$$

where the actual cut-off angle $\kappa_{a}$ depends on the current aircraft pitch:

$$
\kappa_{a}=\kappa-\theta
$$

Calculation of this cut-off point in advance enables the pilot to judge when to flare the aircraft. A view from the cockpit of the flare initiation moment is shown in Figure 5(b). The advantages of these longitudinal points on the runway are twofold: errors in judgement have a much smaller impact on the altitude perception and runway width is not a factor. As the runway surface markings are standardized, centerline markings and touchdown zone areas offer pilots the cues to judge longitudinal distances independent of runway width.

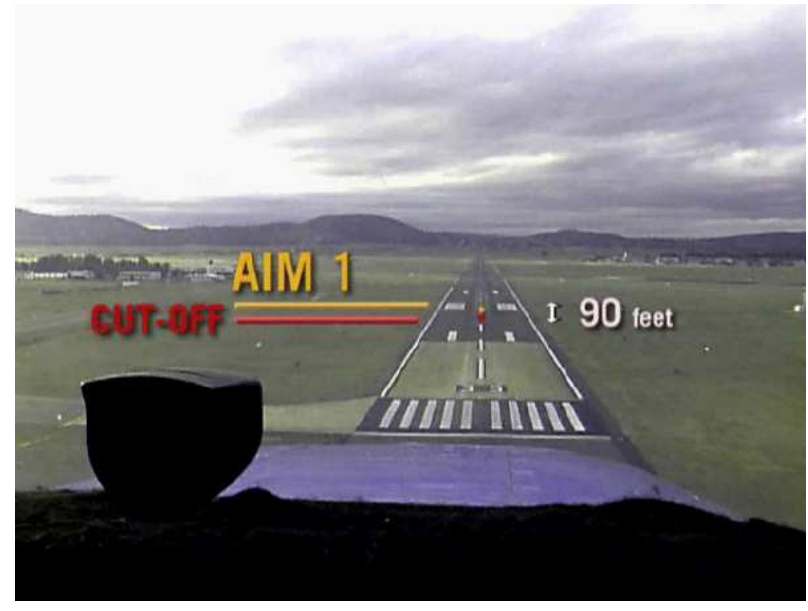

(a) short final

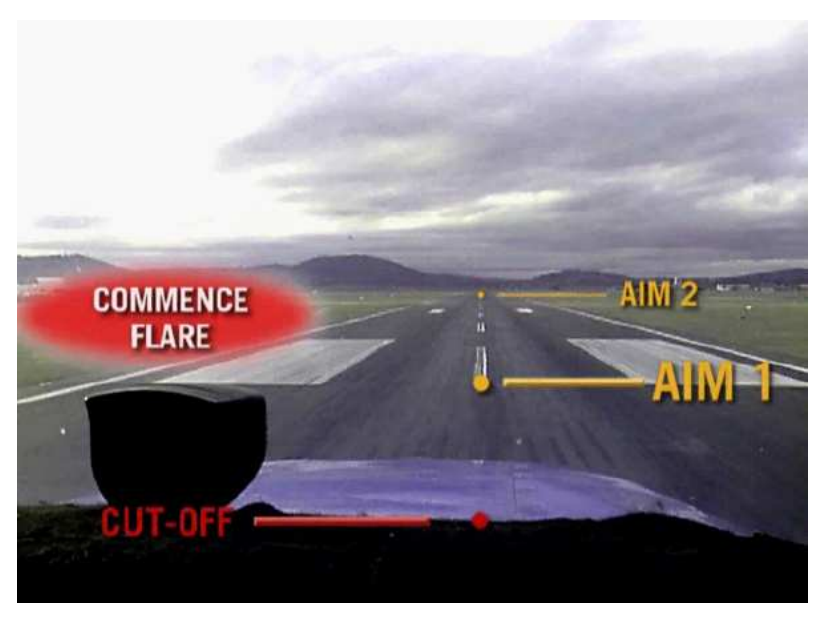

(b) flare initiation point

Figure 5. Aimpoint and flare cut-off cues viewed from Cessna 172 cockpit $^{7}$

\section{Flare execution}

The question remains how to fly the aircraft down close to the desired aimpoint within safety limits after initiating the flare. The Jacobson flare method applies the "Gentle Touch" method" ${ }^{16}$ to guide the aircraft through the flare down to the touchdown point. This method uses the same aimpoint following concept to fly the visual path. What the pilot needs to do upon flare initiation is to make the far end of the runway the new reference aimpoint. The pilot then progressively pitches the aircraft in such a manner that over 3-4 seconds, a same sight picture is attained (as flying a constant aimpoint path angle prior to the flare). This is illustrated by Figure 6 as seen from the cockpit.

The far end of the runway provides a distant and stable reference point that allows for smooth control. This method enables the aircraft's flight path to converge to the new aimpoint and descends the aircraft gently until the wheels touch the runway. This aimpoint shift profile is shown in Figure 7. 


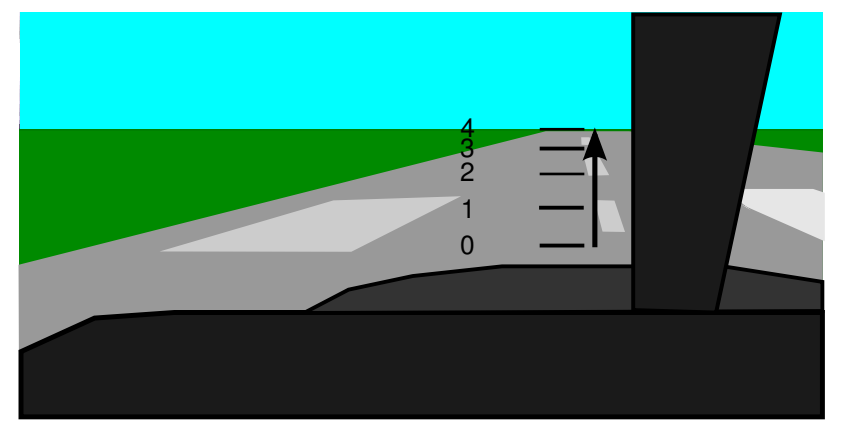

Figure 6. Aimpoint shift per second towards end of the runway

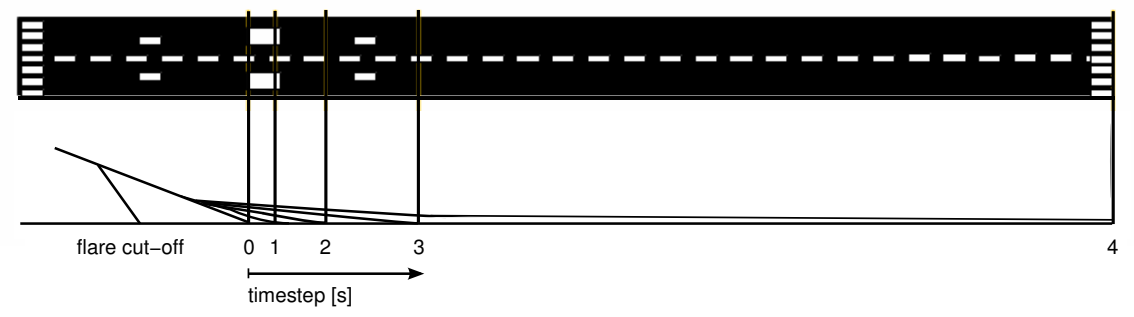

Figure 7. Aimpoint shift after flare initiation ${ }^{7}$

\section{Offline simulation results}

\section{Automatic flare simulation}

The automatic flare control system as described in the previous section was modeled in Matlab/Simulink to provide numerical data for comparison with manual piloted flares. The required aircraft dynamics of the Cessna Citation 500 aircraft are implemented in CitAST. ${ }^{17}$ For analyzing the flare with pitch command and throttle inputs, a symmetric model of the aircraft is sufficient. Therefore the symmetric model was selected. The model was subsequently trimmed and linearized to the same initial conditions as the theoretical example. An automatic throttle using a pure gain controller kept the airspeed constant during the approach. Upon reaching the flare point, a switch toggled the auto throttle from airspeed hold mode to idle cutback. A rate controller ensured realistic spool down times for the engines were simulated.

The desired versus actual altitude profile for an automatic flare is shown in Figure 8. Touchdown occurs at 24.7 meters after the glide slope transmitter at an altitude of $0.5 \mathrm{~m}$. This is due to the aircrafts landing gear extending this distance below the center of gravity.

Time responses of the relevant aircraft state variables are given in Figure 9. It can be seen that the desired exponential flarepath is achieved by reducing the flightpath angle to zero. This has a direct impact on the vertical speed, which also exponentially reduces to zero, achieving a smooth landing. The aircrafts true airspeed drops linearly as a result of pitching up and the autothrottle cutting back to idle.

The energy profile of the flare is shown in Figure 10. The potential energy of the aircraft reduces characteristically to the exponentially decaying altitude profile. The potential energy has a very small contribution to the total energy profile having a $1.9 \%$ maximum ratio of potential energy to total energy at the start of the flare. The reduction of kinetic energy is reflected by the airspeed decrease during the flare. Overall, energy does not present a good indicator of flare execution since the altitude and airspeed differences are too small and occur in a very short time span. 

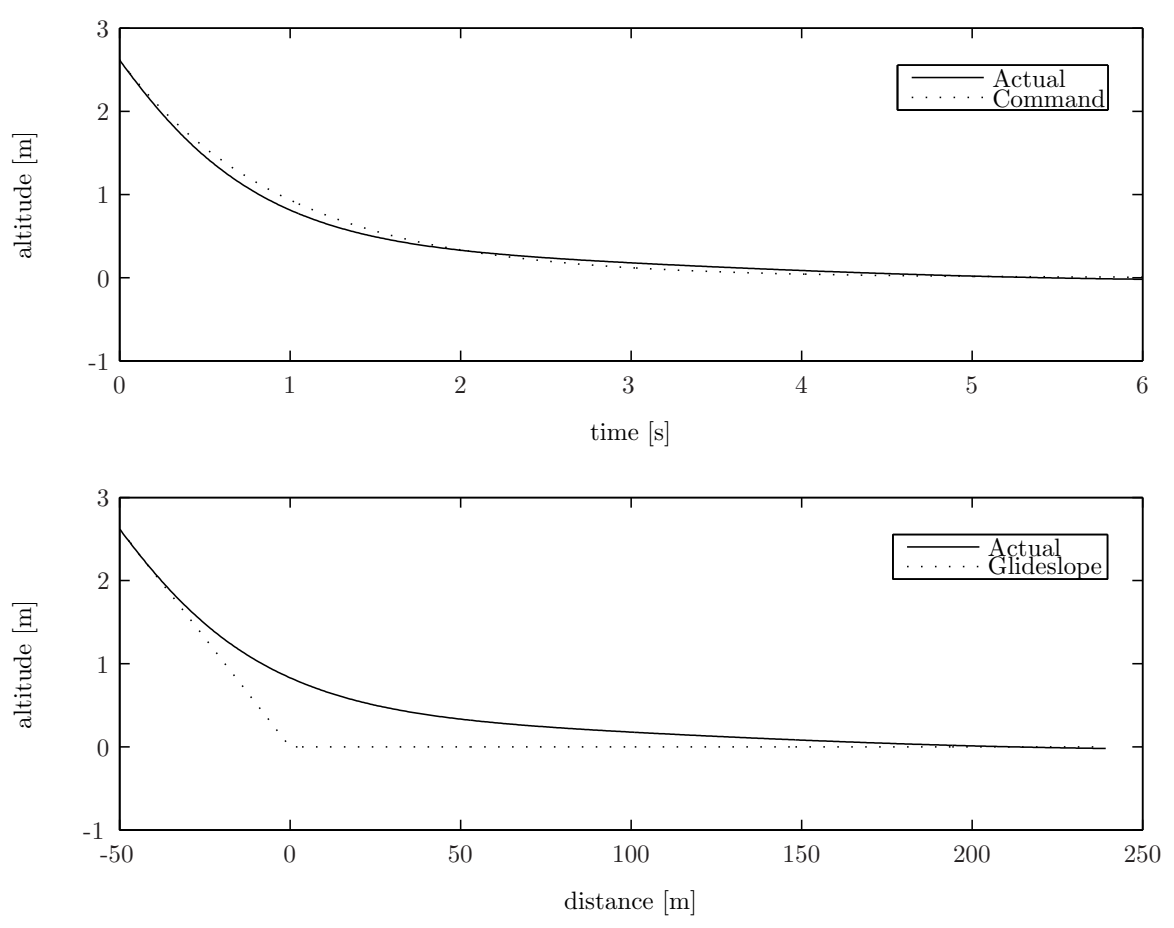

Figure 8. Altitude profile of automatic flare simulation
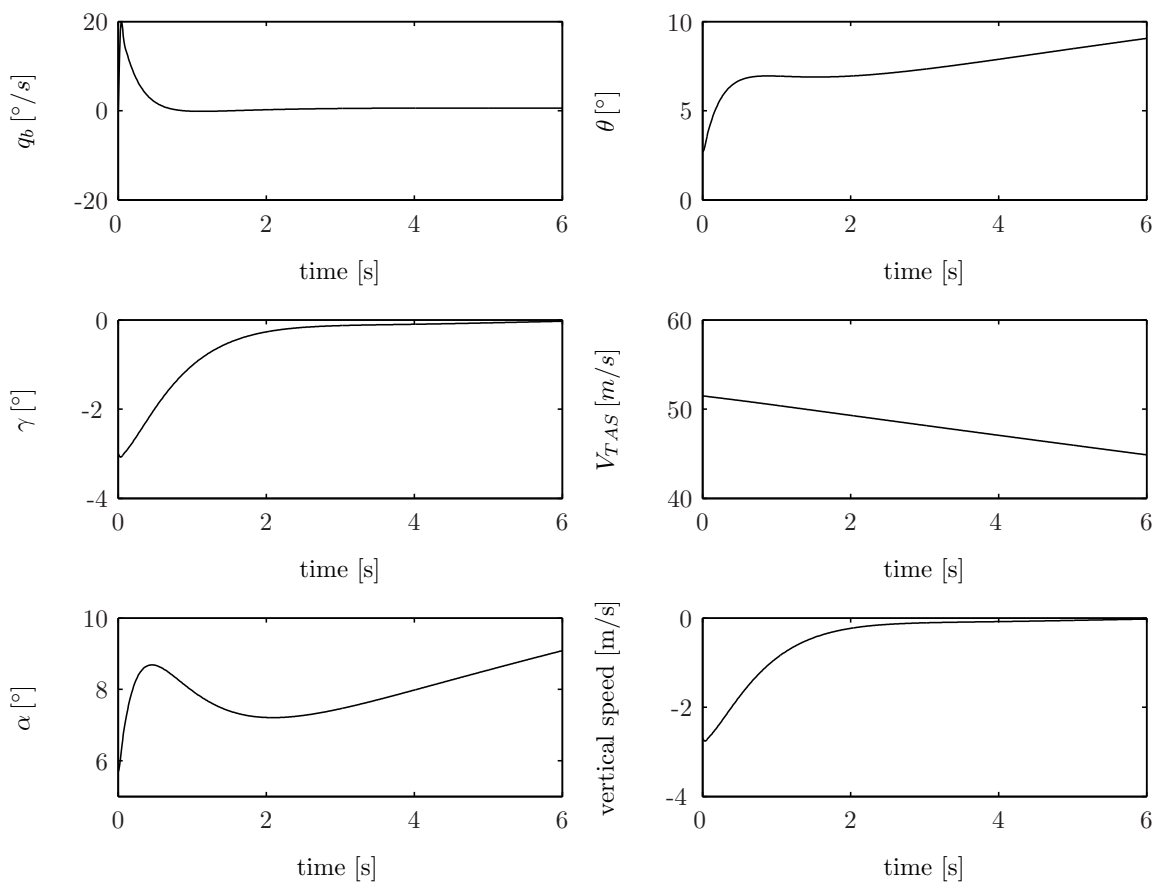

Figure 9. Time reponses of automatic flare simulation 

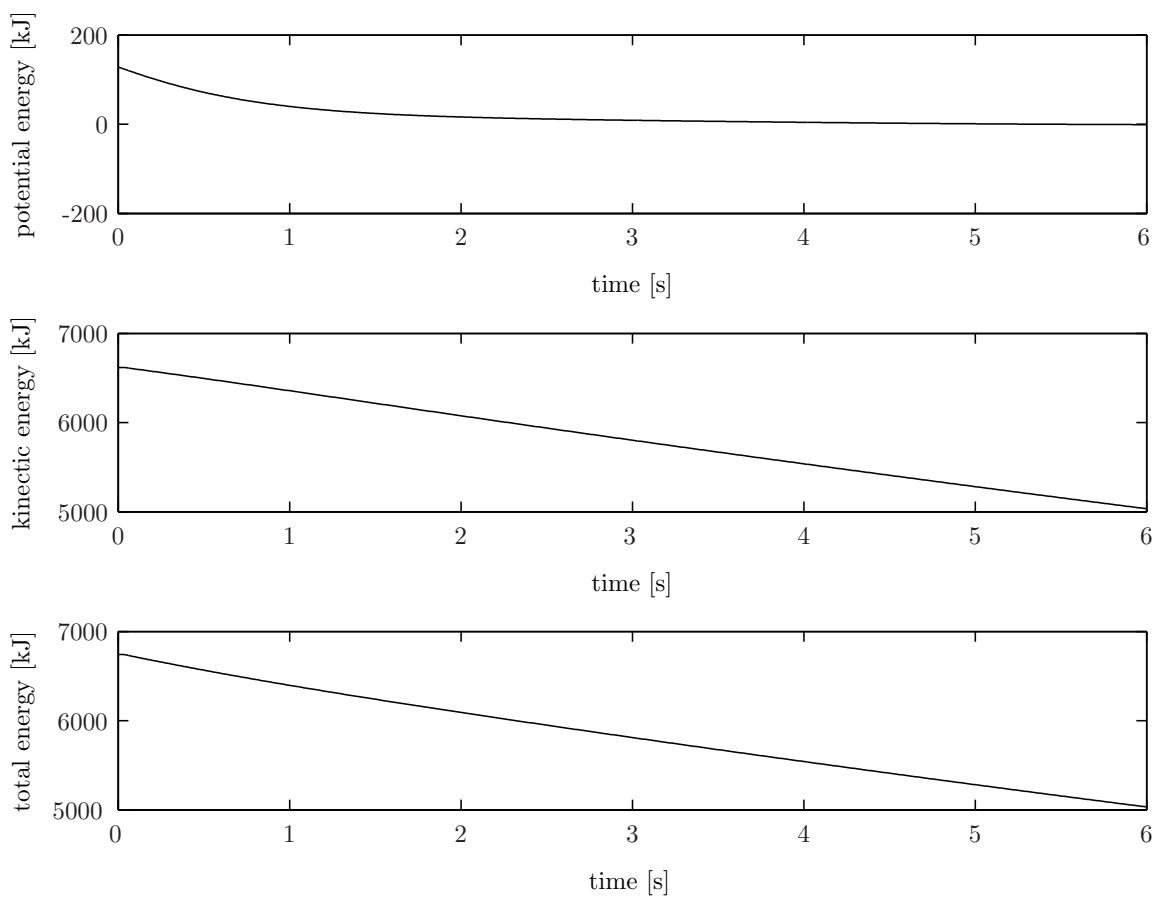

Figure 10. Energy plots of automatic flare simulation

\section{Jacobson linear simulation}

A simulation of the Jacobson flare was performed using the same linear, aircraft model for the automatic flare simulation. Additional blocks to model the visual cues and control of the aircraft were also created.

The aircraft starts at an initial altitude of 100 feet. The initial ground distance is adjusted so that the ILS antenna is exactly on the glideslope beam. The flare controller then flies the aircraft down by keeping the aimpoint angle with the glareshield constant. With the aircraft trimmed, it requires no further control input until the flare initiation point. The aircraft time responses are shown in Figure 11.

At $t=9.54 \mathrm{~s}$, the cut-off point is reached and the flare is started. The Jacobson flare is implemented with a pitch angle controller: the aircraft is commanded to a $\theta=5.7^{\circ}$ nose high end state, in contrast to the automatic flare controller which commanded the flight path angle to zero (Figure 9). The aircraft's vertical speed is reduced back to $-1.2 \mathrm{~m} / \mathrm{s}$ on touchdown.

The altitude profile is shown in Figure 12(a). It can be seen that the ILS antenna matches the glideslope beam prior to the flare and that main gear touchdown occurs at $t=11.68 \mathrm{~s}$. The altitude profile as a function of ground distance is given in Figure 12(b). It shows main gear touchdown was 16.8 meters beyond the glideslope emitter, much closer than what the automatic flare controller achieved.

To control the flare after initiation with the "Gentle Touch" method, the aimpoint was shifted to the end of the $2000 \mathrm{~m}$ long runway via a polynomial function. The angle between the pilot's eye and the current aimpoint, $\lambda$, was calculated for every timestep. This is shown in Figure 13. The aiming angle which the controller tries to keep constant is obtained by subtracting the current cockpit cut-off angle $\kappa_{a}$ from $\lambda$. These visual angles are shown in Figure 14.

On a perfect glideslope prior to the flare, $\lambda=3^{\circ}$, which matches the initial flight path angle. At flare initiation, the aimpoint shifts towards the end of the runway causing the aiming angle to become larger as seen in Figure 14. The flare controller corrects this deviation by pitching the aircraft's nose up to track the same lower aiming angle. Manual tweaking of the controller resulted in it being able to transition to the new aimpoint at the end of the runway in 2.5 seconds which yielded a successful landing. 

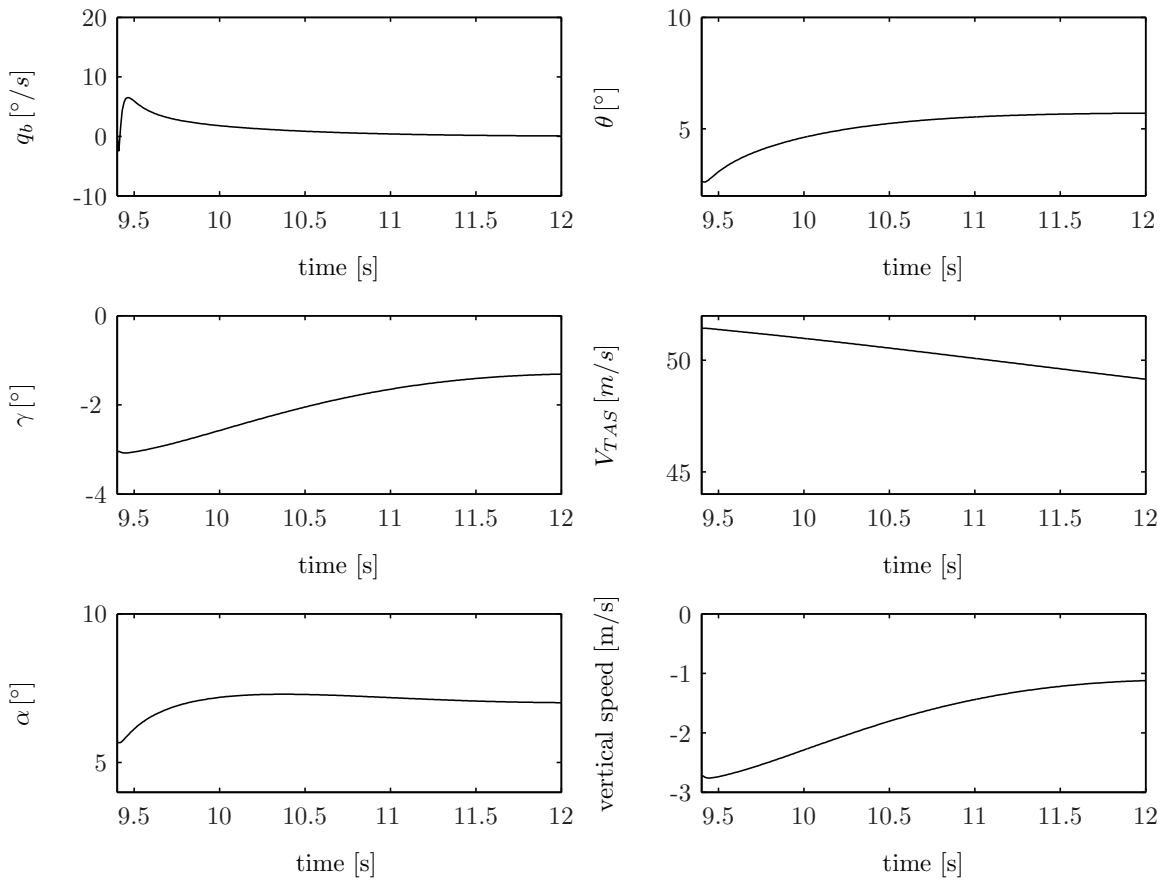

Figure 11. Time responses of Jacobson flare simulation

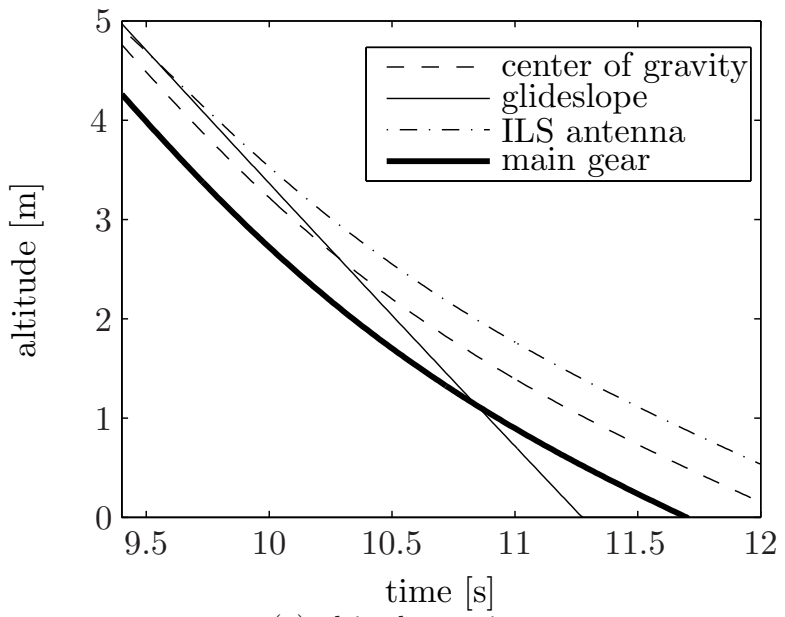

(a) altitude vs. time

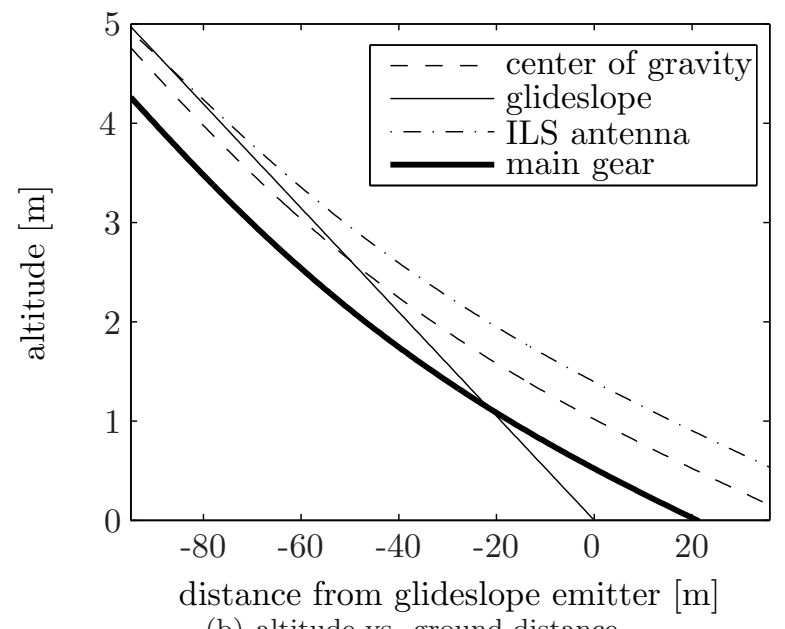

(b) altitude vs. ground distance

Figure 12. Altitude profile of Jacobson flare simulation

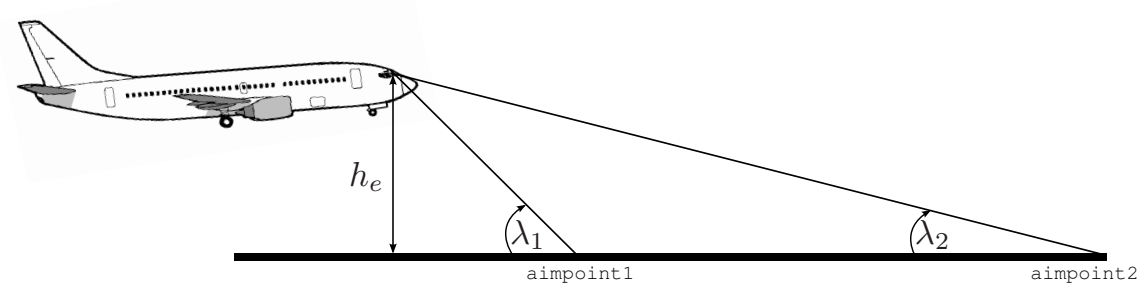

Figure 13. Angle between pilots eye and aimpoint $\lambda$ example for two aimpoints 


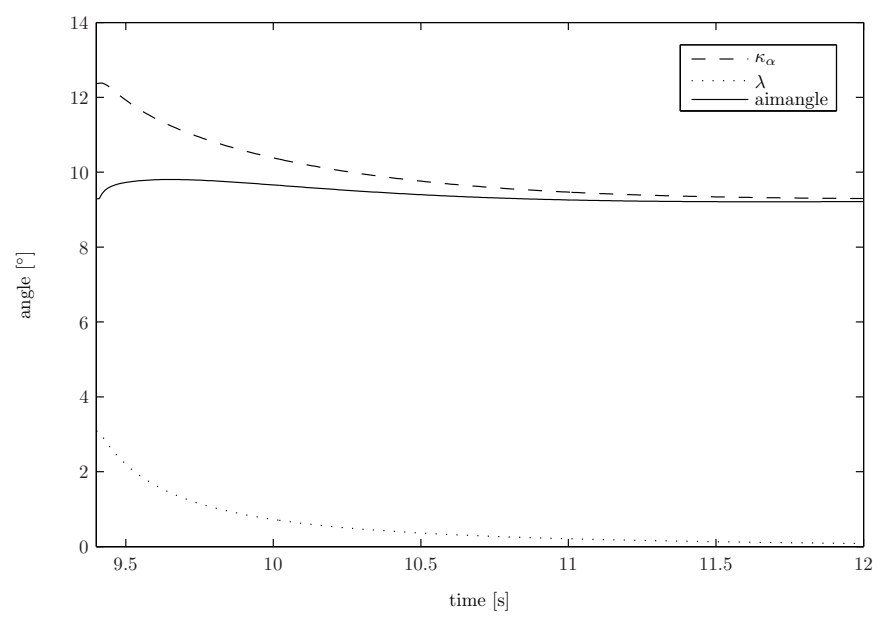

Figure 14. Visual aimpoint angles of Jacobson flare simulation

\section{Sensitivity analysis}

Since the controller is designed to fly the Jacobson flare for the optimal glideslope, a sensitivity test was conducted for off-design conditions by varying the initial altitude of the aircraft and fixed distance before the glideslope emitter at the start of the simulation.

Vertical sink rates upon touchdown are shown in Figure 15(a). The vertical sink rate increases until $2.0^{\circ}$ glideslope and reduces to the lowest value at $3.3^{\circ}$. It increases again as the glideslope increases to $4.5^{\circ}$. These touchdown vertical sink rates can be considered safe as the absolute values were within safe limits with a maximum sink rate of $-1.3 \mathrm{~m} / \mathrm{s}$.

Figure 15(b) shows a linear relation between initial glideslope and touchdown position relative to the glideslope emitter. The lower the approach, the shorter the touchdown point is on the runway. Vice versa, a higher approach resulted in a longer touchdown position.

Figure 15(c) shows that a higher initial glideslope yields higher pitch values. This confirms that a shallow approach requires much less pitch up as compared to a steep approach. A steep approach needs more pitch to bleed off the excess energy.

The simulated Jacobson flare controller was designed for the $3^{\circ}$ perfect glideslope approach and did not manage to replicate the 3-4 seconds of pitch up to match the aimpoint at the far end of the runway after flare initiation. With steeper approaches, it would aggressively pitch the aircraft's nose and already reduce the aiming angle in 2 seconds. In real life, manually flown ILS approaches that are one degree off glideslope are visually noticeable and considered poor airmanship. The Jacobson flare is flexible and compensates the flare timing for the approach, however it can not salvage poorly flown approaches.

\section{The Interfaces}

\section{A. Basic SVS display}

A schematic of the basic SVS display with the support cues is shown in Figure 16(a). The displays viewpoint origin is at the pilots eye reference point. The field of view (FOV) is $30^{\circ}$ to enable better tracking performance during the final approach and reduce the minification level. ${ }^{18}$ The synthetic generated terrain has ground and runway textures. The SVS display consists of pitch bars, a flight path marker (FPM), airspeed tape to the left, altitude tape and vertical velocity indicator to the right of the display.

\section{B. Enhanced SVS display}

Four cues are added to support the pilot in timing the flare initiation as well as executing the flare to: aiming line, flare initiation line, eye path predictor (EPP) and angle of attack bracket. The enhanced SVS display 


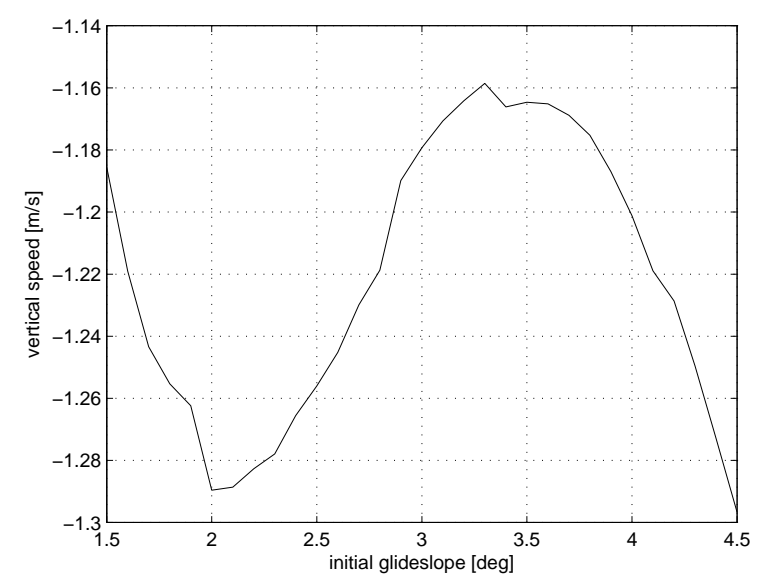

(a) touchdown vertical speed

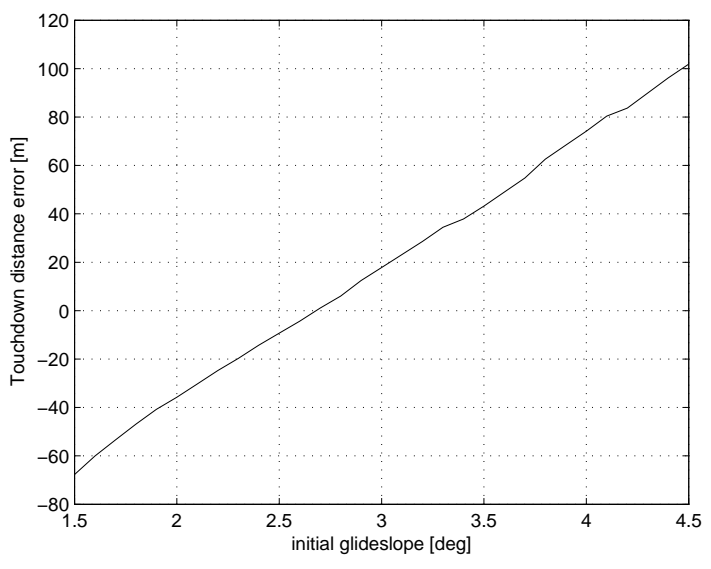

(b) touchdown distance from aimpoint

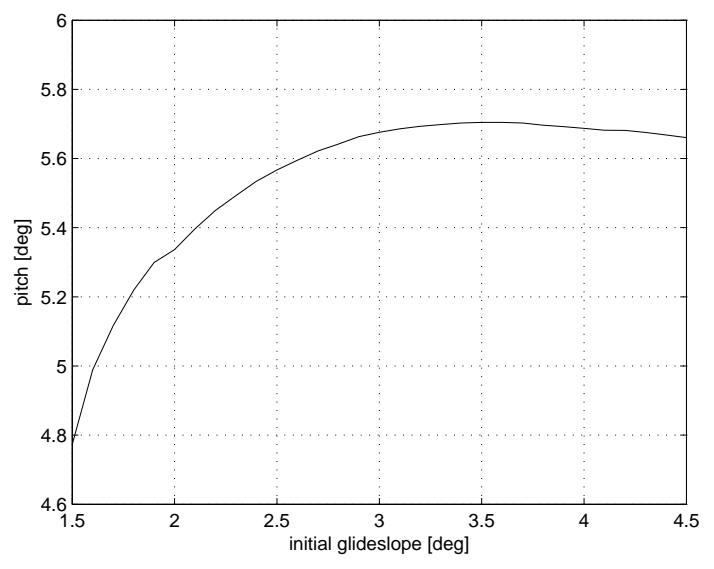

(c) touchdown pitch angle

Figure 15. Sensitivity analysis of Jacobson flare simulation 


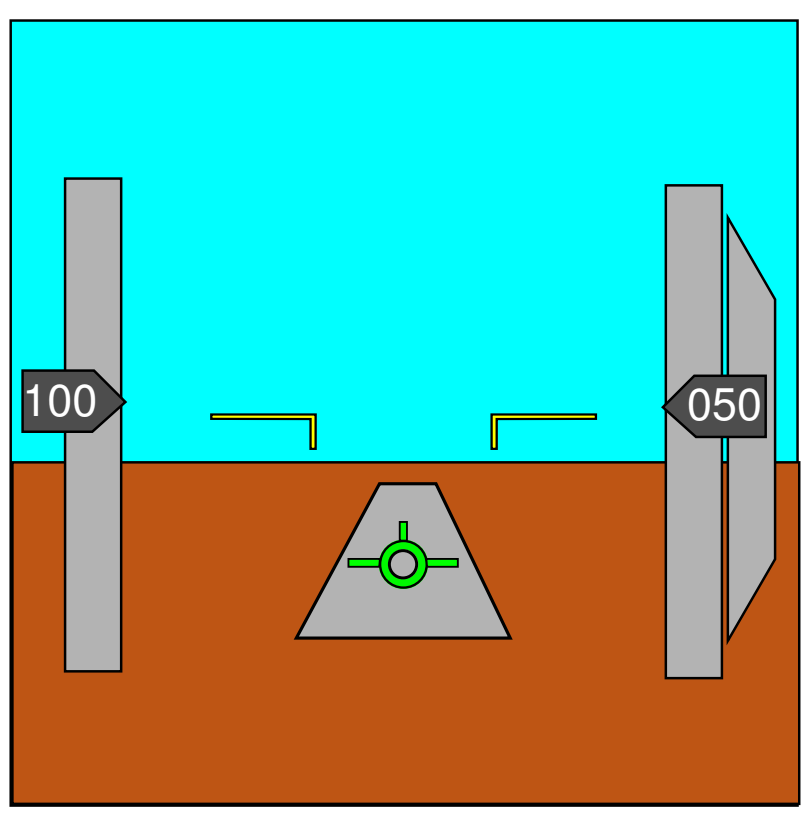

(a) basic

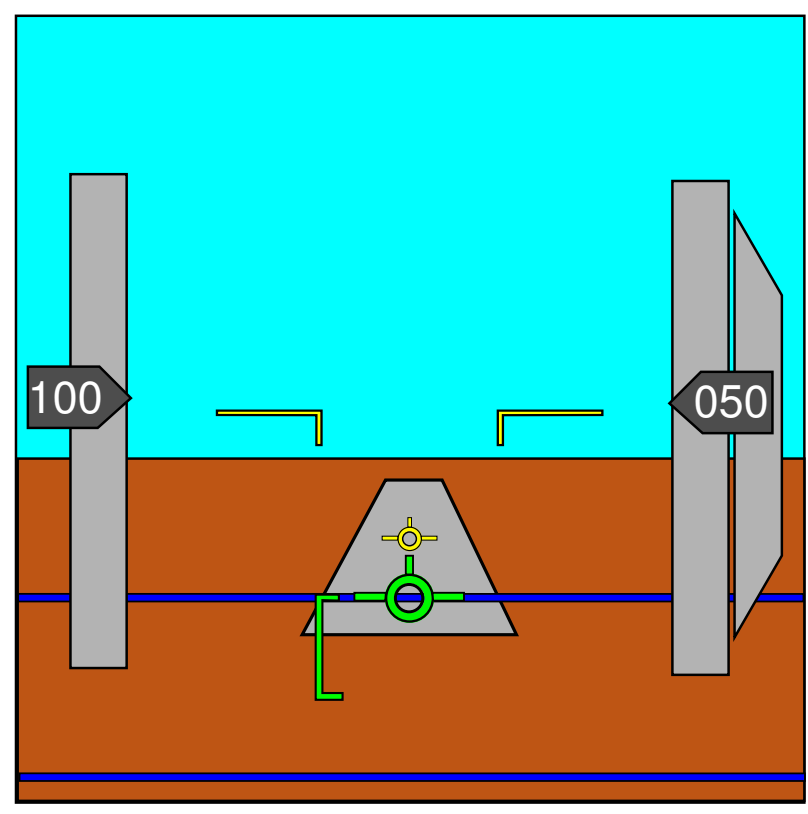

(b) enhanced

Figure 16. Artist impression SVS diplay concept

with the support cues is shown in Figure 16(b).

\section{Aiming line}

The Jacobson flare method uses an aiming point on the runway prior to flare initiation. With a FPM available on the SVS display, the pilot steers the FPM over the desired aiming point. An aiming line spanning horizontally across the runway is used instead to give a better visualization and is shown as the top blue horizontal line in Figure 16(b).

\section{Flare initiation line}

The flare cut-off point can be determined by using the FOV of the SVS display instead of the normal cockpit lower cut-off angle. It is a horizontal spanning line. When it moves to the bottom and is out of view on the SVS display, then the flare initiation point for the pilot has been reached. The lower blue horizontal line in Figure 16(b) represents this flare initiation line.

\section{Eye path predictor}

To support the pilot after flare initiation, an EPP is added to the display. The EPP shows the future predicted vector for the pilot's eye path corresponding with the Jacobson flare method. It is similar to a regular flight path predictor FPP that represents aircraft's future FPM for the center of gravity. It projects the FPP but is shifted forward to geometrically correspond with the eye path which is ahead of the aircrafts center of gravity path. The smaller, yellow aircraft silhouette symbol in Figure 16(b) depicts the EPP. The prediction time for the EPP is a constant 3.5 seconds. This is in concurrence with Robson's "Gentle Touch" method. Giving the prediction of the eye path in 3.5 seconds simplifies the task of controlling the flare. The pilot only has to pitch the aircraft upon flare initiation such that the EPP is placed on the far end of the runway and keep it there. The prediction time for the EPP is similar to the optimum prediction time for a FPP during the flare. Pilot gain behavior was constant after 3 seconds of FPP prediction time and the optimum time was 5 seconds in relation to performance. ${ }^{10}$ It must be noted that with 5 seconds of FPP time, pilots had to judge when to flare by themselves. 


\section{Angle of attack bracket}

The angle of attack is used as a parameter to control the aircraft on final approach. It offers a direct measure of stall margin independent of aircraft weight and responds earlier than airspeed to stick and throttle inputs. ${ }^{19}$ It also offers a single display point regardless of flap settings and gross weight for straight-in approaches in particular. ${ }^{20}$ The pilot maneuvers the flight path with pitch and adjusts power for angle of attack. This angle of attack margin on a SVS display is visualized by using C-shaped bracket as can be found on the the Heads-Up Display (HUD) of military jets (F-16, F/A-18). The bracket is referenced to the horizontal centerline (left wing) of the FPM symbol. A $0.5^{\circ}$ hysteresis should be used to prevent flickering. ${ }^{21}$ The angle of attack bracket (also referred to as energy bracket on the F/A-18) and its usage together with the FPM is shown in Figure 17.

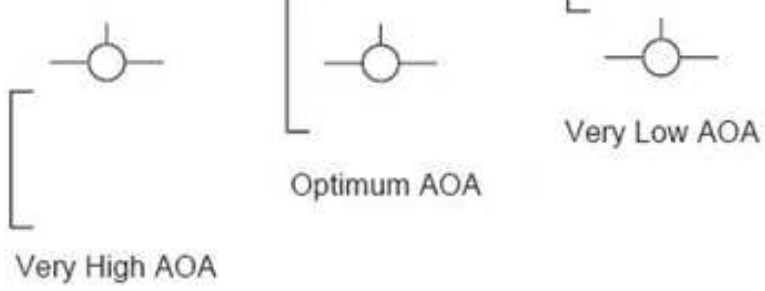

Figure 17. The angle of attack bracket

\section{Experiment}

\section{A. Method}

To validate the effectiveness of the new additional flare support cues on a SVS display, the experiment comprised three display types: (1) the pilot has outside visuals but only conventional instruments (this set a baseline performance), (2) a basic SVS display with no outside visuals, (3) an enhanced SVS display with without outside visuals. The three display type cases are presented in Table 1.

Table 1. The three experiment cases

\begin{tabular}{ccc}
\hline \hline Case & Display & Outside Visual \\
\hline 0 & PFD & Yes \\
1 & SVS & No \\
2 & SVS Flare & No \\
\hline \hline
\end{tabular}

The outside visual scenery consists of a textured, asphalt runway with ICAO compliant precision runway markings. The surrounding terrain is a representation of the Rotterdam airport (EHRD) area. There are however, no 3D objects such as buildings or trees in the scenery. A picture of the outside visuals can be seen in Figure 18.

The format of the basic SVS display is depicted in Figure 19. The Primary Flight Display is exactly the same as this but has the synthetic runway omitted when flying with outside visuals enabled. The synthetic runway is rendered with the same ICAO precision markings as the outside visuals.

In this format the pilot can perceive the following flight status parameters:

1. the aircraft Indicated Airspeed (in knots) by means of a speed tape,

2. the aircraft bank angle, 


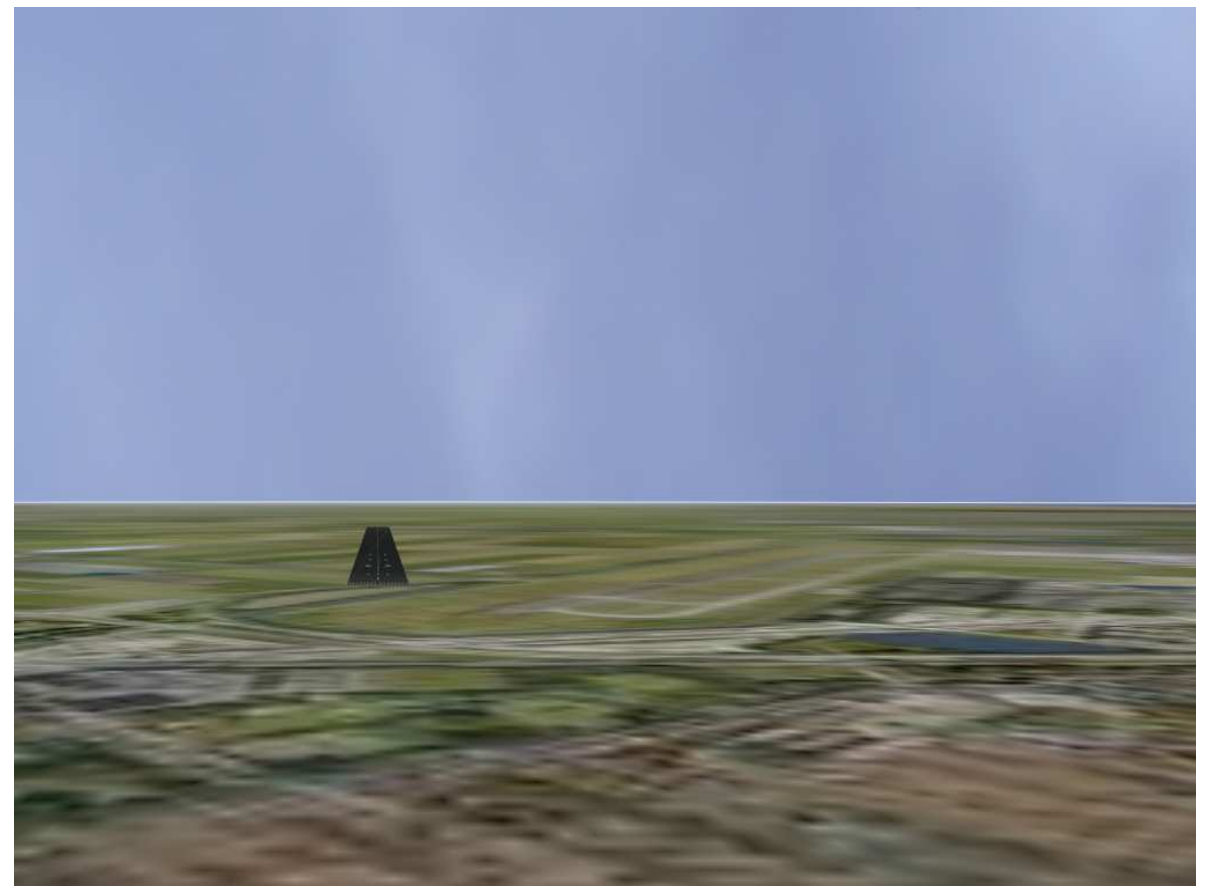

Figure 18. The outside visual scenery

3. the aircraft altitude (in feet) by means of the altitude tape,

4. the pitch attitude by means of the pitch ladder,

5. the horizon line,

6. the direction of the aircrafts motion by means of a flight path vector symbol,

7. the aircraft reference symbol,

8. the current heading of the aircraft by means of a compass rose,

9. the vertical airspeed (in feet per second).

Besides the basic SVS features and synthetic runway, the enhanced display incorporates three additional cues. The EPP cue was discarded because during testing it was found that turbulence made the symbol bounce around making it not usable. Making the prediction time longer to values commonly used during cruise would nullify its purpose during the flare. Applying a turbulence filter was considered but this would introduce a significant system lag considering the critical short time of the flare maneuver. Pilots have to perform the flare without this cue by raising the aircraft's flight path vector up to the end of the runway or slightly below the horizon in 3 seconds time. The remaining enhancements should still provide the pilot with clear, concise information during the very short final approach and flare. The enhanced symbology are highlighted in Figure 19(b) and comprise of:

a. the aiming line,

b. the flare line,

c. the alpha bracket.

\section{Subjects}

Eight professional glass-cockpit airline pilots participated, with an average age of 30 years $(\mathrm{STD}=3.99)$ and an average experience of 3,554 $(\mathrm{STD}=2694.96)$ flight hours. Eight novice pilots also participated with an average age of 25 years $(\mathrm{STD}=2.20)$. In total, $(8+8=) 16$ subjects participated in the experiment. 


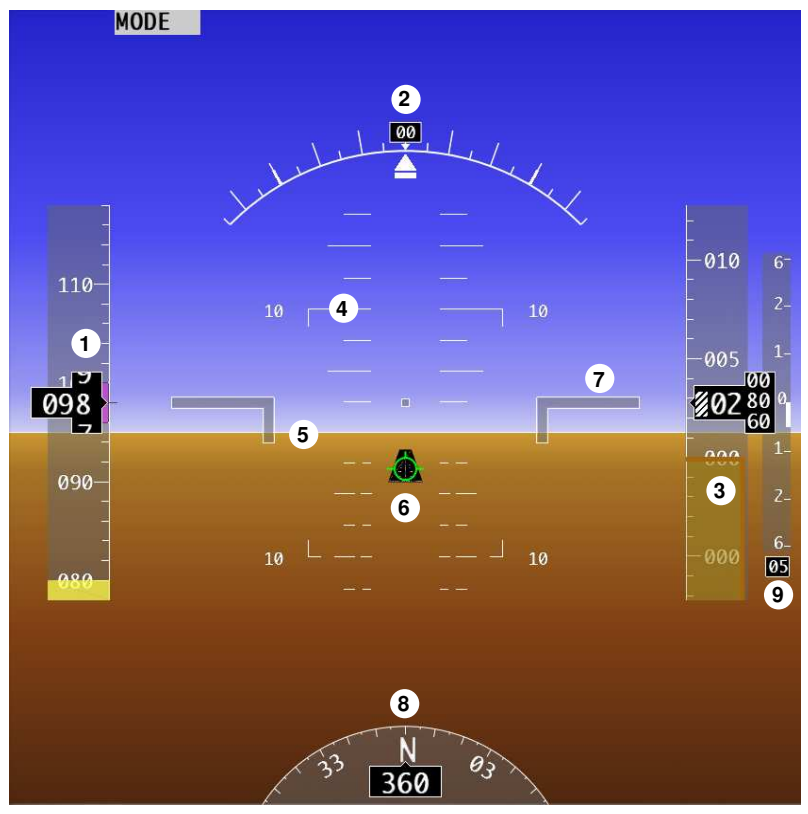

(a) basic

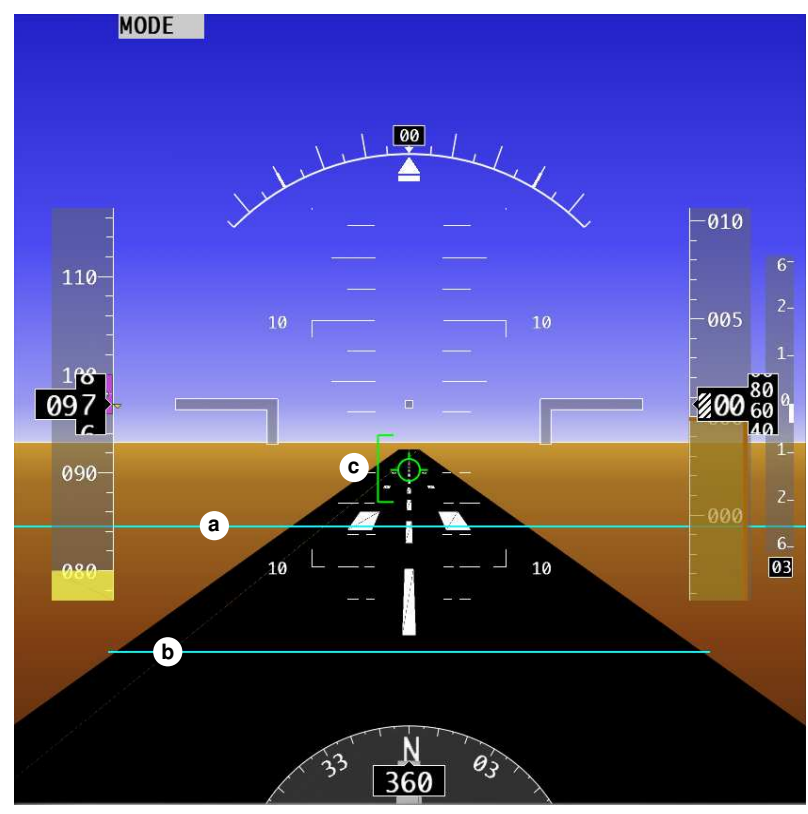

(b) enhanced

Figure 19. SVS display interfaces

\section{Independent variables}

a. Within-subject variables. The experiment had two within-subject variables: the display configuration (DISP) and initial glideslope condition (GS). DISP had three levels: primary flight display, basic SVS and enhanced SVS (SVS Flare). Outside visuals were only provided with the primary flight display (PFD), all SVS displays were without any outside visuals. GS had three levels: on glideslope, one dot low and one dot high.

b. Between-subject variables. Once pilots have learned how to fly the enhanced display, it was hypothesized that they would assimilate this technique and have it applied to the other displays. Therefore, the display order variable (DO) was conceived to test this. Each pilot group was split into two to accommodate the DO which had two levels: PFD-SVS-SVS Flare and SVS Flare-SVS-PFD. The first level meant all training and experiment runs were performed using basic instruments with outside visual first. Then pilots moved on to the SVS display and finally to the enhanced display. The second level was this display order but swapped.

\section{Scenarios}

Since this experiment focused on the fundamentals of the flare, only one scenario was tested: a straight-in approach and landing. The usage of low, center and high GS conditions was combined with placing the aircraft at either 30,33 or 37 seconds before touchdown occured. This ensured pilots could not memorize the runs and cheat by counting the time elapsed until flare initiation was appropriate.

\section{Instructions}

The pilots were instructed to fly a stable approach to the designated aimpoint and flare the aircraft. The objective was to achieve touchdown close to the aimpoint while ensuring safety (touchdown sink rate $<2.0$ $\mathrm{m} / \mathrm{s}$ ). Pilots were briefed to conduct a missed approach instead of attempting to salvage a landing if any problems occured. 


\section{Apparatus}

The experiment was conducted in the Human-Machine Laboratory located at the Faculty of Aerospace Engineering of the Delft University of Technology. The laboratory consisted of an experiment cabin with a fixed-base simulator and a simulation control area. The pilot was seated in an adjustable real co-pilots chair in a darkened experiment cabin while the experimenter stayed outside the experiment cabin in the simulation control area. Directly in front of the pilot was a 18 LCD screen which was used to display the PFD or SVS to the pilot. To the left of the pilot, in the center of the cabin mockup, was another 18" LCD screen, which showed the engine display. The pilot controlled the aircraft via a right-handed, electrohydraulic, servo-controlled, passive side stick (elevator and aileron). In this experiment, the side stick was configured to simulate an ordinary passive manipulator; stick position determined direct output to the aircraft's control surfaces. Trim controls and rudder pedals were not used during this experiment. The aircraft was always preset in the landing configuration so pilots did not have to operate the flaps and gears. The throttle quadrant was available on a center pedestal but only the throttle levers were used. The outside visuals were projected via a single projector in front of the pilot seat.

\section{Aircraft characteristics and experiment conditions}

The aircraft model used in the experiment was a non-linear, six degrees of freedom model of the Cessna Citation 500. The aircraft started trimmed for a three degree approach but on different glideslope positions before the runway. The aircraft was preset in the landing configuration with 40 degrees flaps, gear out and on an approach speed of 98 knots indicated airspeed. During flight, trimming was not possible and the rudders were not used. The aircraft did have a yaw damper enabled. The atmosphere was modeled according to the International Standard Atmosphere with light turbulence but no wind.

\section{Dependent measures}

The dependent measures were:

a. Performance. Touchdown position and sink rate were the two main parameters used to grade the performance of the experiment. Touchdowns close to the aimpoint and soft landings were considered to be successful achievements.

b. Self assessment. Self assessment measured whether the pilot had a good awareness of his actions during landing. Pilots were requested to mark their estimated touchdown position after each run. This was then compared to the actual touchdown point. Pilots were also asked to self grade each run. This had five levels: very poor - poor - average - good - very good. To compare self grading across the pilots, the results were transformed into z-scores. The self assessment form is shown in Figure 20.

c. Workload. Pilot workload was measured using a NASA TLX rating sheet after each run. To compare workload scores between pilots, this data was also transformed into z-scores.

d. Safety. Safety was measured by counting the number of crashes (excessive sink rates). Any missed approaches due to safety concerns conducted by the pilot would also be duly recorded.

\section{Final questionnaire}

A questionnaire regarding preferences and opinion on the displays was given to the pilots after they have finished all experimental runs. Pilots were asked to indicate what cues they used during specific phases of the landing. They also had to rank the displays by providing a preference to use which display during a phase of the flare.

\section{Experiment design}

Both the professional and novice pilots were each divided into two groups: each half (4 professional pilots +4 novices) operated the SVS display with support cues first and the other half (4 professional pilots +4 novices) used it as last. The straight-in approach scenario was flown with three initial glideslope conditions in a randomized order per display type. Each pilot flew $(9 \times 2=) 18$ runs per display totalling 


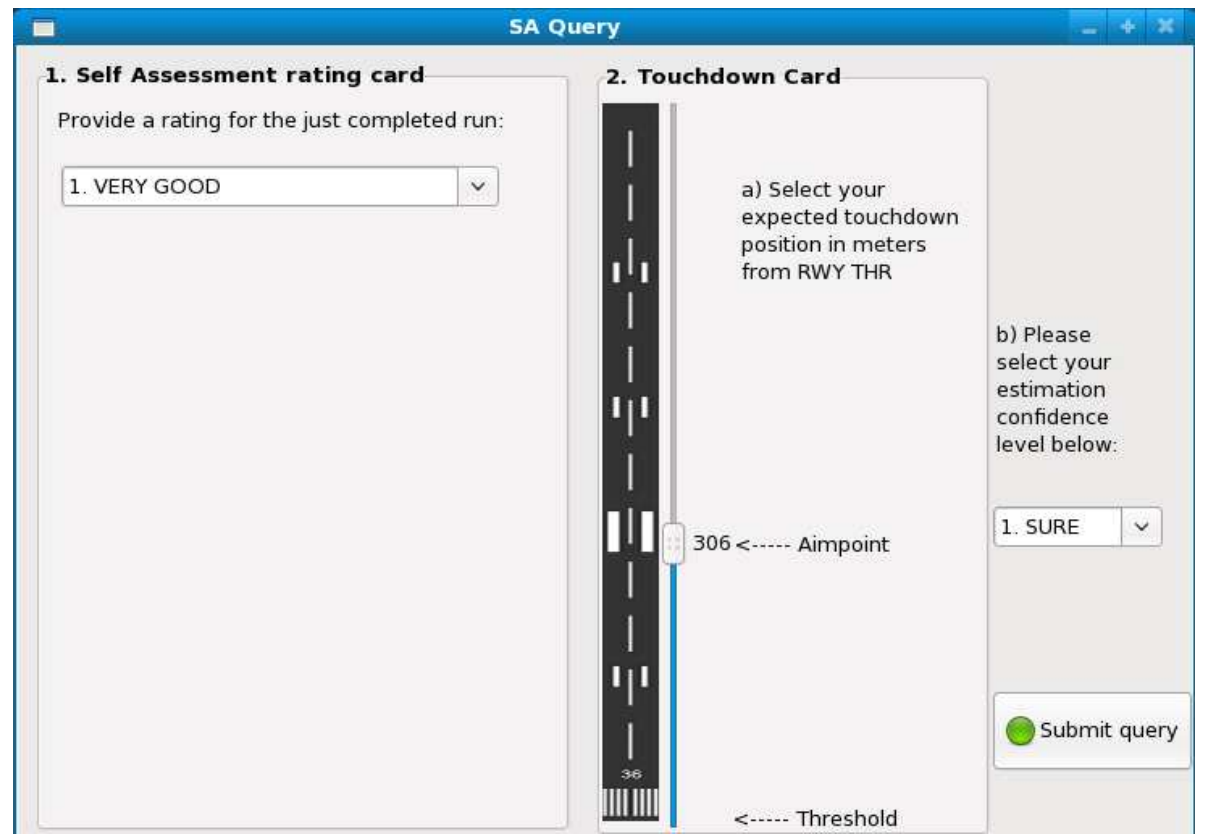

Figure 20. Self assessment query

$(18 \times 3=) 54$ measurement samples per pilot. Therefore, the professional and novice pilot groups each generated $(54 \times 8=) 432$ samples. This led to a total of $(432 \times 2=) 864$ samples for the entire experiment.

\section{Procedure}

The experiment procedure consisted of two phases. The first phase was considered a training phase in which the pilot got accustomed to landing with the various displays in 10 runs. The second phase was the measurement phase consisting of 18 runs to be flown per display type with short breaks in between. Each pilot was divided into a specific group which had a display order to perform the experiment. The first group performed visuals landings first. This was followed by basic SVS landings without any outside visuals. Finally this group was briefed about the SVS enhancements after which they performed landings using the enhanced display. Group 2 performed the experiment in reverse order starting with the SVS enhancements and ending with visual landings. A single experiment run lasted around a minute and pilots had to complete a self assessment query after each run. A NASA TLX workload rating was administered after each display type. Finally, the experiment was concluded with a final questionnaire.

\section{B. Hypotheses}

The enhanced SVS display with flare support cues was hypothesized to yield the best pilot performance results as it presents the pilots with a quantifiable cue to commence the flare and clear instructions on how to control the flare until touchdown. Touchdown awareness was also expected to be the highest with the support cues. The basic SVS display was assumed to be the worst for the performance and situational awareness due to smaller visual angles and less visual cues as compared to the outside visuals.

Flight safety was expected to be the best with the enhanced display as its cues supports pilots in judging when or not to conduct a missed approach. The effect of the display order was expected to be significant resulting in better performance and situational awareness when the pilots started with the enhanced display compared to starting with the classic instruments and outside visuals first. Pilot workload was assumed to be the highest for the basic SVS display and the lowest for the SVS display with support cues. 


\section{Results}

A statistical analysis of the experiment data output was done by a repeated-measures Analysis of Variance (ANOVA) with mixed within- and between-subjects variables. Neither crashes nor missed approaches occurred and all landings were within the safety limits.

\section{A. Performance}

\section{Professional pilots}

Analysis of touchdown locations for the professional pilot group showed that display type (DISP) was not significant $(F(2,92)=1.897, p=0.156)$. Initial glideslope condition (GS) proved to have a highly significant influence on touchdown position $(F(2,92)=8.688, p<0.01)$. Contrasts showed pilots achieved touchdowns closer to the aimpoint when starting established on the glideslope. However, there was no significant effect between being initially 'too low' or 'high' (Figure 21(a)). DISP $\times$ GS was not significant $(F(4,184)=0.729, p=0.573)$. The display order (DO) in which the two groups performed the experiment was significant $(F(1,46)=5.601, p=0.022)$. This revealed a learning effect that the second group of pilots demonstrated by achieving overall touchdown positions closer to the aimpoint. This is shown in Figure 21(b).

The vertical speed at touchdown was highly significant depending on DISP $(F(2,92)=7.521, p<0.01)$. The softest landings were achieved based on outside visual, followed by the enhanced display. Shown in Figure 21(c), both SVS displays had more scatter in vertical speed compared touchdowns with outside visuals. GS was not significant $(F(2,92)=2.454, p=0.092)$ and neither was $\operatorname{DISP} \times \operatorname{GS}(F(4,184)=$ $0.466, p=0.0761)$. DO also had no significant effect on the touchdown sinkrate $(F(1,46)=0.034, p=0.854)$.

\section{Novice pilots}

The novice test subjects achieved opposite touchdown position results as compared to the professional pilots. DISP was highly significant $(F(2,92)=14.512, p<0.01)$, GS was not significant $(F(2,92)=$ $1.754, p=0.179), \mathrm{DISP} \times \mathrm{GS}(F(4,184)=2.968, p=0.021)$ was significant and DO was not significant $(F(1,46)=2.051, p=0.159)$. Outside visual landings were the furthest from the aimpoint with contrast revealing the difference to either the basic or enhanced display to be highly significant (Figure 22(a)). However, there was no significance between the basic and the enhanced display.

Mauchly's test indicated that the assumption of sphericity had been violated for effects on vertical speed due to DISP $\left(\chi^{2}(2)=20.518, p<0.05\right)$ and DISP $\times \operatorname{GS}\left(\chi^{2}(9)=36.928, p<0.05\right)$. Therefore degrees of freedom were corrected using Greenhouse-Geisser estimates of sphericity $(\epsilon=0.732)$ for DISP and $(\epsilon=0.751)$ for DISP $\times$ GS. DISP was highly significant $(F(1.464,67.342)=12.104, p<0.01)$, GS not significant $(F(2,92)=0.233, p=0.793)$ and DISP $\times$ GS also not significant $(F(3.003,138.132)=0.251, p=$ $0.861)$. Softest landings were again achieved with outside visuals with no significant differences between the SVS displays (Figure 22(b)). DO was highly significant $(F(1,46)=7.443, p<0.01)$ : the group that first performed outside visuals first achieved softer landings overall (Figure 22(c)).

\section{B. Self assessment}

\section{Professional pilots}

The z-score self assessment grades for both the professional and novice pilots had no significance due to any dependent measures at all. The error in estimated and actual touchdown position for the professional pilots was only borderline significant for DO $(F(1,46)=4.136, p=0.048)$. The first group starting out on outside visuals had better estimations of their touchdown locations. However, this could not be attributed to any DISP or GS effects.

\section{Novice pilots}

The novice pilots produced more distinct results: DISP was highly significant $(F(2,92)=7.369, p<0.01)$. Contrasts showed that outside visuals proved to be the most difficult in order to estimate the touchdown 


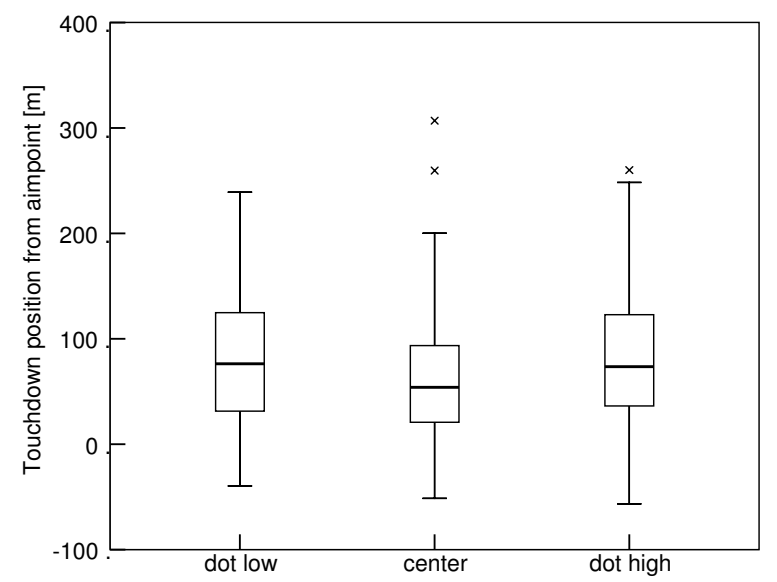

(a) Touchdown position vs. glideslope

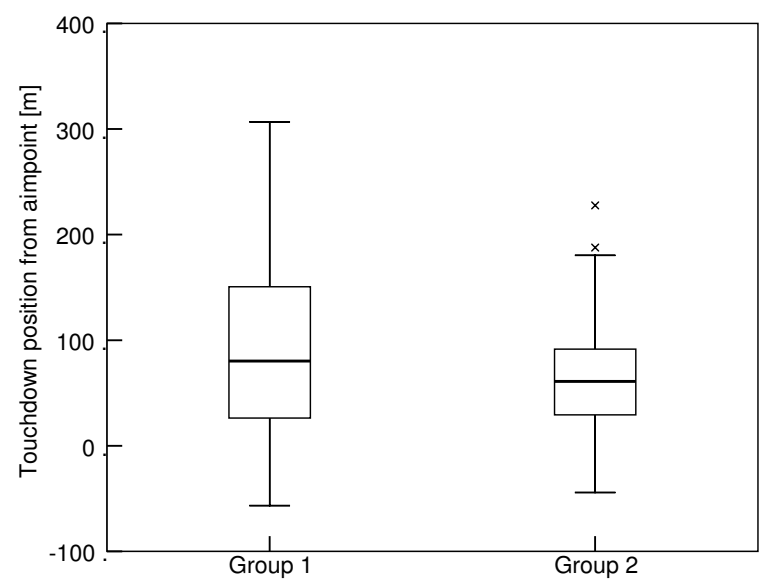

(b) Touchdown position vs. display order

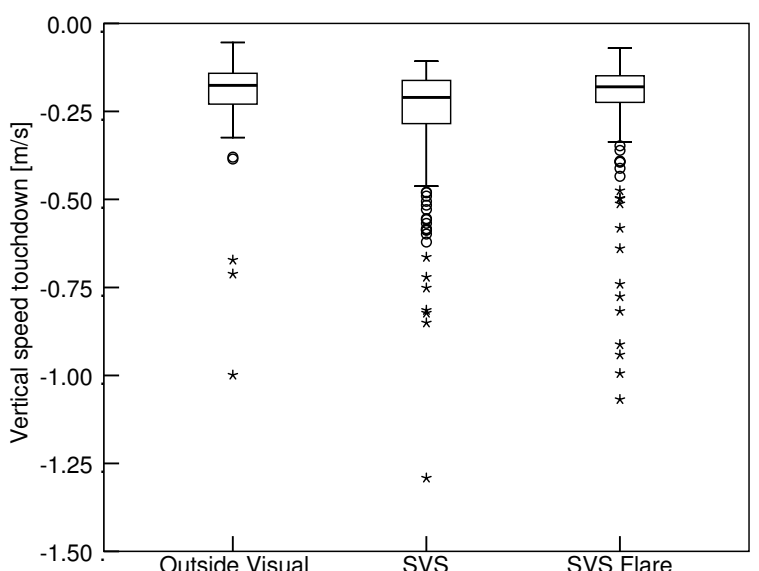

(c) Vertical speed vs. display type

Figure 21. Boxplots of the highly significant performance measures for the professional pilot group 


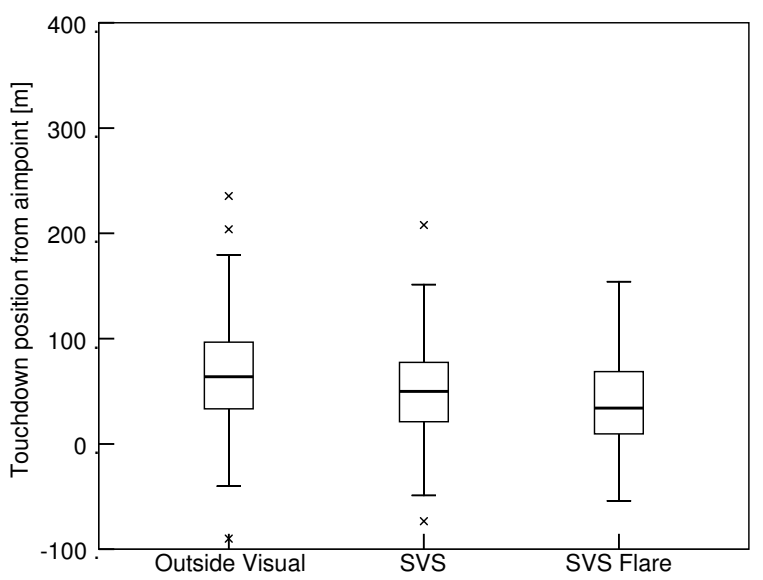

(a) Touchdown position vs. display type

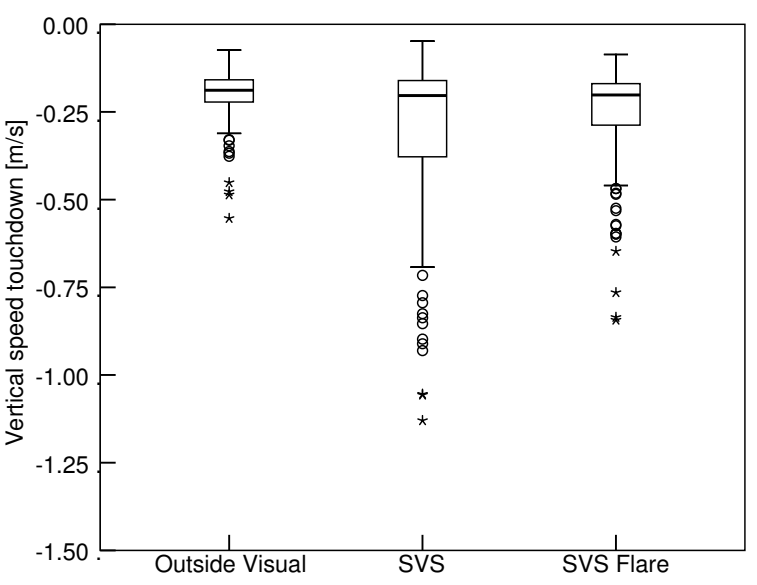

(b) Vertical speed vs. display type

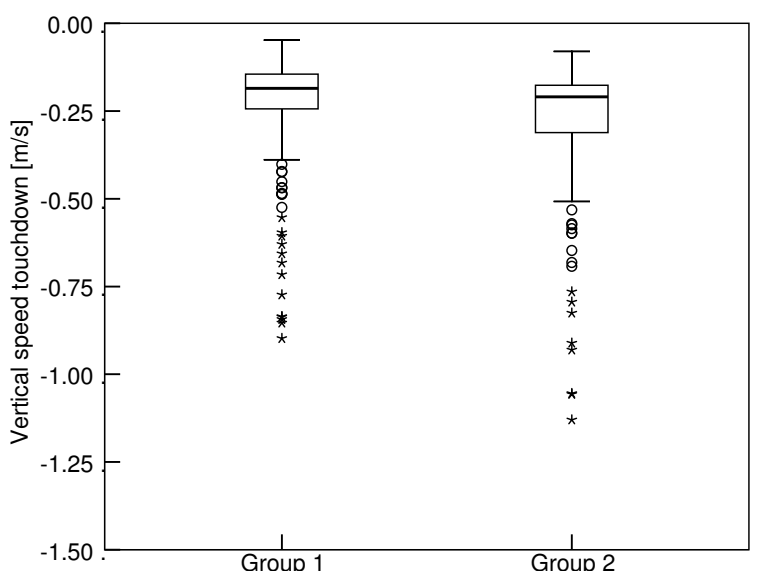

(c) Vertical speed vs. display order

Figure 22. Boxplots of the highly significant performance measures for the novice group 
position. The two SVS displays allowed better estimation although there was no significance between them (Figure 23(a)). GS $(F(2,924)=2.994, p=0.055)$ was borderline significant but inspection of GS showed that there was no significance between being on initial glideslope or not. DISP $\times \operatorname{GS}(F(4,184)=2.482, p=0.045)$ was significant and this interaction revealed that higher initial GS caused larger estimation errors for SVS displays. DO was highly significant $(F(1,46)=13.594, p<0.01)$. Contrasts showed that the group starting with the enhanced display were able to achieve better estimated means of error that were twice as small as compared to the group starting with outside visuals (Figure 23(b)).

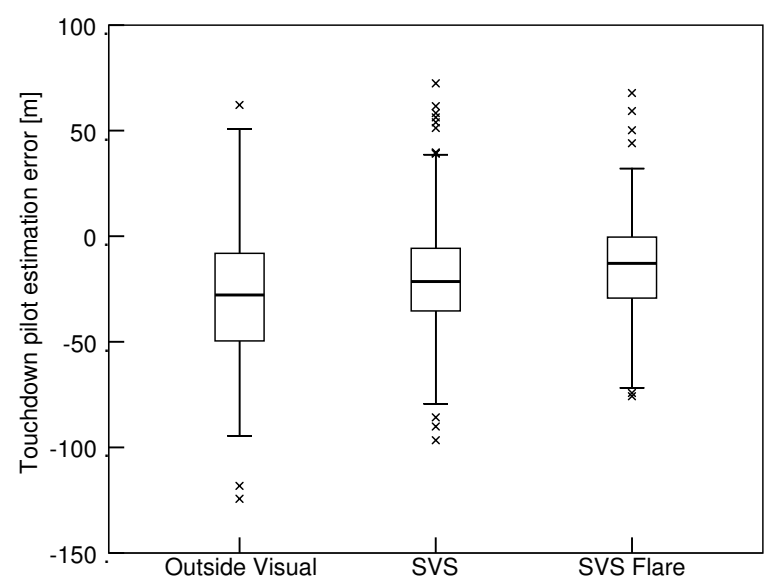

(a) Touchdown estimation error vs. display type

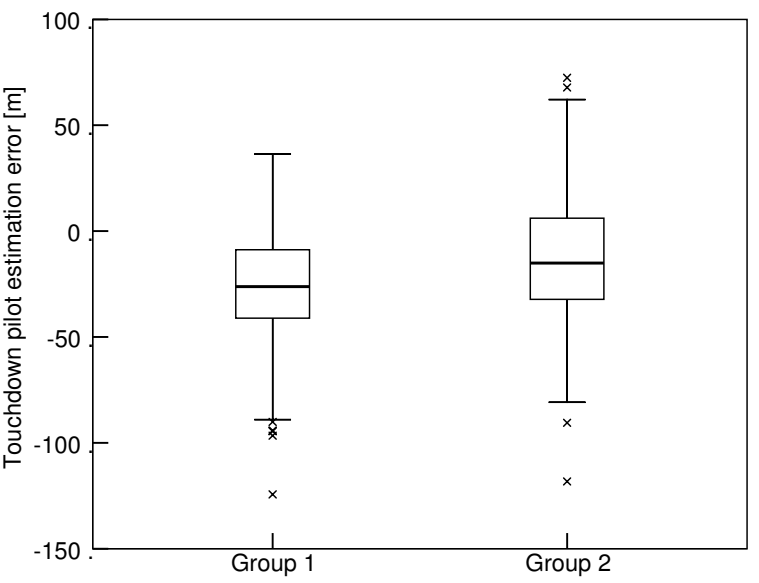

(b) Touchdown estimation error vs. display order

Figure 23. Boxplots of the highly significant self assessment measures for the novice group

\section{Workload}

The analysis of the administered NASA TLX ratings did not reveal any significant effect for either test groups. Therefore, this test method was not able to confirm if the workload, as hypothesized, differed between the displays. The pilots did comment that although the enhanced display led to more control activity, there was no extra physical workload involved. It also benefited their path tracking accuracy by offering a clear path error cue; pilots were making minute adjustments from further out which they were not able to do with the other displays because the angular error was not prominent enough.

\section{Correlation analysis}

\section{Professional pilots}

The data was checked whether it was normally distributed; this was not the case so a non-parametric Spearman correlation test was done. It showed that touchdown position had a significant negative correlation to the sink rate $(r=-0.116, p=0.016)$. This indicated long landings would result in firmer touchdowns. The pilots were settling down at higher sink rates to salvage touchdown position when the aircraft was coming in long. There was also a significant negative correlation between touchdown position and estimation error $(r=-0.277, p<0.01)$. This would indicate that landing or floating too long made it harder to estimate the touchdown position. Touchdown position and self assessment grade were significantly correlated $(r=0.295, p<0.01)$. This is obviously due to the experiment goal that landing closer to the aimpoint equals a better performance. Vertical speed had a significant correlation to the touchdown estimation error $(r=-0.263, p<0.01)$. This meant hard landings made it more difficult for pilots to judge touchdown since contact with the ground happened too rapidly and abruptly. Vertical speed also had a significant negative correlation to the self assessment $(r=-0.100, p=0.039)$. This indicated that pilots gave higher ratings for soft landings, which would correspond to landings further from the aimpoint. This reflected that pilots were really aiming for precision landings as stated by the objective. Finally, the touchdown estimation error 
had a significant correlation to the self assessment grade $(r=0.167, p<0.01)$ : better estimations concurred with good landing self ratings.

\section{Novice pilots}

The novice group had a significant positive correlation for touchdown position and sink rate $(r=0.187, p<$ 0.01). This is opposite to the professionals, novice pilots made softer landings whenever touchdowns were long. This implied they considered it a more important factor for their control strategy. Touchdown position was similar with a significant negative correlation $(r=-0.610, p<0.01)$ for estimation error and a significant correlation $(r=0.136, p<0.01)$ for self assessment score. Vertical speed had both a significant negative correlation with estimation error $(r=-0.206, p<0.01)$ as well as for self assessment $(r=-0.163, p<0.01)$. Another contrast with the professional pilots is that there was no significant correlation between touchdown estimation error and self assessment grade.

\section{E. Final questionnaire}

For the entire landing, pilots ranked the enhanced display as the best followed by the basic SVS display. The enhancements and landing technique was found to be intuitive and easy to perform. Outside visuals were the least favored. Pilots also stated their dislike for flying off a projected image on a wall in front. The lack of surrounding visuals was also stated to be an issue by not making use of peripheral vision available to judge velocity when close to the ground. For flare initialization, the display rankings were very pronounced: pilots clearly favored the enhanced display and they stated that it was the only one with a definite initiation cue. With the basic display or outside visuals, pilots indicated they used an equal share of altimeter, runway geometry, runway markings and distance to aimpoint as cues to start the flare. The basic SVS display was ranked higher than outside visuals.

Flare control after initiation did not provide any distinct preferences, all displays were ranked exactly the same. This was because pilots used the same method of shifting the flight path to slightly below the horizon after flaring. The only difference is that pilots had to judge the flight path using optical flow on outside visuals compared to both SVS displays that provided a direct FPV symbol. Pilots, however, did favor FPV representation with the consensus that flying using a visual aimpoint could easily be degraded during adverse weather and reduced visibility conditions. Although this experiment was of a visual nature, half of the pilots indicated they missed the radar altimeter callouts that they are using during real-life approach and landings. They thought audio callouts would make a good addition as an extra crosscheck. The enhanced display symbology gave half of the pilots a better synoptic view on a small area which reduced the workload of monitoring flight parameters. However, the other half of the pilots found this compactness of information to be a possible source of 'tunnel vision' prior to the flare. Some pilots suggested to omit the flare line until very short final to reduce clutter on approach. A trend line or command cue to support post flare initiation would be appreciated by some pilots. The angle of attack bracket was considered more useful during the approach. Pilots suggested that an option to toggle it on or off would be beneficial to reduce clutter during the flare.

\section{Discussion}

The Jacobson method did provide a better comprehension of the flare by breaking it down into two stages and defining distinct visual cues. Enhancing the basic SVS display with these cues has aided pilots in providing clear information on when and how to flare. Display type did not affect touchdown position for the professional pilots as it did for the novice group. The airline pilots were accustomed to handle larger, heavy transport aircraft and as such may not have acquired enough familiarity with the lighter jet to maximise their landing performace. Pilots did appear to follow instructions and use the displays as briefed, but when they mistimed the flare they seemed to apply control corrections using their own methods and experience. This could have been an obstacle impeding more distinct results. On the contrary, the novice group made closer touchdowns without outside visuals. Minimal flight experience apparently led to a more objective assimilation of flare technique once taught. But it could also be explained that minimal experience on outside visuals may have resulted in poor landings because they were less familiar with the sight picture and 
associated visual stimuli. But no significant result between the basic and enhanced display failed to prove the direct benefits of the extra symbology. Although touchdown position was not influenced by display type, display order did. Armed with the prior knowledge of the technique to flare with the enhanced display, pilots were more likely to make precise landings. This proves that it could be used as an educational tool to familiarize pilots with the flare during training and potentially reduce the number of flight training hours required.

Both SVS displays were inferior to outside visuals regarding touchdown sink rate. This demonstrated that for control after flare initiation, outside visuals offered more pronounced cues to control sink rate which the SVS lacked due to minification. This implied that minification and especially the reduced field of view of the SVS displays were detrimental to performance. ${ }^{22}$ More research into this area is needed to offer pilots the additional support needed with SVS: the conceptual flight path predictor made usable for turbulence could provide this support.

Pilot self assessments did not reveal differences between the displays. This was due to precision runway markings being available on all displays, making it easy to remember the touchdown position relative to the markings. Pilots did comment that the aircraft model used was very easy and forgiving. For future experiments, it is recommended to use a large, heavy transport aircraft representative of airliners. This ensures results will not be influenced due to varying pilot adeptness levels to the aircraft. The dynamics of a larger, heavy transport aircraft will also make any effects of flare control more pronounced. The novice pilots that started with the enhanced display did have better touchdown position awareness. This can be deduced from the fact that more consistent landings were made that required little to no adjustment of the touchdown position estimate.

No evidence on workload differences could be found between the displays. The flare is still an intensive task which requires critical timing such that even the enhancements, simplifying the parameters to perform the flare, did not make it less demanding. This is expected to remain unchanged since the flare remains the most crucial maneuver of any flight. Correlation between touchdown position and sink rate between the professional and non-professional groups revealed a difference in performance measure prior to the flare. The professional pilots were aware and consciously made the choice of getting a touchdown position closer to the aimpoint at the cost of a higher sink rate. The novice group was more concerned with landing the aircraft at low sink rates to avoid any crashes. Due to experience, the professional pilots were well aware of the practical value range for safe sink rates and took the decision to optimize for touchdown position. This corresponds to the objectives as stated in the experiment briefing.

The most useful interface feature that the enhanced display offered was the flare line. This provided an unambiguous cue for pilots to anticipate the initiation of the flare. However, pilots still had to judge when the flare line would disappear and time the flare such that pitch rotation occurs simultaneously. Removing this judgement factor and enabling a countdown cue could remove anticipation errors and improve flare initiation consistency. The aiming line provided a larger visual cue during the approach and allowed for more precise control to get the aircraft to the flare point to allow optimal use of the flare line. This did come at a cost by an increase in pilot control activity, pilots were constantly aligning the flight path with the aiming line all the way before starting the flare. The aiming line should be of higher significance when landing on runways of smaller width. An experiment with varying runway widths is suggested to validate for more results.

\section{Conclusions}

The Jacobson flare proved to be a viable method to design landing support cues for synthetic vision display augmentation. The experiment showed that the enhanced display supported pilots in achieving better touchdown performance compared to the basic display. However, it was still inferior to outside visuals in touchdown sink rates. The strong learning curve effect of the enhanced displays suggested that this could be used as an excellent tool for flight training, offering pilots a better understanding of the flare maneuver and in a shorter time. 


\section{References}

${ }^{1}$ Le Ngoc, L., "European Personal Air Transportation System (EPATS) study: Cockpit avionics \& human machine interface requirements," Technical Report NLR-Memorandum ASAS-2007-066, National Aerospace Laboratory, Amsterdam, 2007.

${ }^{2}$ Mena, M. P., "Enhanced Vision and Synthetic Vision in the Next Generation Air Traffic System," 2007.

${ }^{3}$ Higdon, D., "Seeing Is Believing: Synthetic vision takes spreads through business aviation," World Aircraft Sales Magazine, 2006, Reprinted online: http://www.avbuyer.com/PDFs/Synthetic\%20Vision\%20Systems.pdf.

${ }^{4}$ Parrish, R. V., Busquets, A. M., Williams, S. P., and Nold, D. E., "Evaluation of Alternate Concepts for Synthetic Vision Flight Displays With Weather-Penetrating Sensor Image Inserts During Simulated Landing Approaches," Technical Report NASA/TP-2003-212643, Langley Research Center, National Aeronautics and Space Administration, Hampton (VA), 2003.

${ }^{5}$ Borst, C., Mulder, M., van Paassen, M. M., and Mulder, J. A., "Theoretical Foundations of an Ecological Synthetic Vision Display," AIAA Guidance, Navigation, and Control Conference, Chicago (IL), August 15-18, , No. AIAA-2009-5982, 2009 .

${ }^{6}$ Blakelock, J. H., Automatic Control of Aircraft and Missiles, Wiley, New York (NY), 1991, Second Edition.

7Jacobson, D. M., "The Jacobson Flare," CASA 1997 Flight Safety Seminar Series Presentation, 1997, online at http://www.jacobsonflare.com/downloads/3.\%20The\%20Jacobson\%20Flare\%20CASA\%201997\%20Flight $\% 20$ Safety\%20Seminar\%20Series\%20Presentation.pdf.

${ }^{8}$ Mulder, M., Pleijsant, J., van der Vaart, H., and van Wieringen, P., "The effects of pictorial detail on the timing of the landing flare: Results of a visual simulation experiment," The International Journal of Aviation Psychology, Vol. 10, No. 3, 2000, pp. 291-315.

${ }^{9}$ Jump, M. and Padfield, G. D., "Investigation of the Flare Maneuver Using Optical Tau," Journal of Guidance, Control, and Dynamics, Vol. 29, No. 5, 2006, pp. 1189-1200.

${ }^{10}$ Arents, R. R. D., "Predictive Landing Guidance in Synthetic Vision Displays," Graduation Report NLR-TR-2006-467, National Aerospace Laboratory, Amsterdam, 2006.

${ }^{11}$ Palmisano, S., Favelle, S., Prowse, G., Wadwell, R., and Sachtler, B., "Investigation of Visual Flight Cues for Timing the Initiation of the Landing Flare," Technical Report B2005/0121, Australian Transport Safety Bureau, Canberra, 2006.

${ }^{12}$ Jump, M. and Padfield, G. D., "Development of a Nature-inspired Flare Command Algorithm," AIAA Guidance, Navigation, and Control Conference and Exhibit, Hilton Head (SC), August 20-23, , No. AIAA 2007-6769, 2007.

${ }^{13}$ Jacobson, D. M., "Where to Flare?" 1987 Australian Aviation Symposium 'Innovate or Enervate', Canberra, November 18-20, 1987, 1999 revision (version jfmr99d) available on http://www.jacobsonflare.com/downloads/7.\%20The \%20Jacobson\%20Flare\%20Paper\%20-\%20Where\%20To\%20Flare.pdf.

${ }^{14}$ Arents, R. R. D., Groeneweg, J., Mulder, M., and van Paassen, M. M., "Predictive Landing Guidance in Synthetic Vision Displays," AIAA Guidance, Navigation, and Control Conference, Chicago (IL), August 15-18, , No. AIAA-2009-5984, 2009.

${ }^{15}$ Grosz, J., Rysdyk, R. T., Bootsma, R. J., Mulder, J. A., van der Vaart, J. C., and van Wieringen, P. W., "Perceptual support for timing of the flare in the landing of an aircraft," Local applications of the ecological approach to human-machine systems, edited by J. Flach, P. Hancock, J. Caird, and K. Vincente, Lawrence Erlbaum Associates, Hillsdale (NJ), 1995, pp. 104-121.

${ }^{16}$ Robson, D. P., "The Gentle Touch - Landing an Aircraft Safely, Consistently and Well," 1987 Australian Aviation Symposium 'Innovate or Enervate', Canberra, November 18-20, 1987, pp. 129-131.

${ }^{17}$ Borst, C., "CitAST: Citation Analysis and Simulation Toolkit," Technical report, Faculty of Aerospace Engineering, Delft University of Technology, Delft, 2004

${ }^{18}$ Glaab, L., Kramer, L. J., Arthur, T., Parrish, R. V., and Barry, J. S., "Flight Test Comparison of Synthetic Vision Display Concepts at Dallas/Fort Worth International Airport," Technical Paper NASA/TP-2003-212177, Langley Research Center, National Aeronautics and Space Administration, Hampton (VA), 2003.

${ }^{19}$ Cashman, J. E., Kelly, B. D., and Nield, B. N., "Operational use of angle of attack on modern commercial jet airplanes," Aero Magazine, 2000, Reprinted online: http://www.boeing.com/commercial/aeromagazine/aero_12/attack_story.html.

${ }^{20}$ Gee, S. W., Gaidsick, H. G., and Enevoldson, E. K., "Flight evaluation of angle of attack as a control parameter in general-aviation aircraft," Technical Note NASA TN D-6210, NASA Flight Research Center, National Aeronautics and Space Administration, Edwards (CA), 1971.

${ }^{21}$ Newman, R. L., Head-Up Displays: Designing the Way Ahead, Avebury Aviation, Hants, England, 1995.

${ }^{22}$ Field, E. J., Pinney, T. R., van Paassen, M. M., Stroosma, O., and Rivers, R. A., "Effects of Implementation Variations on the Results of Piloted Simulator Handling Qualities Evaluations," AIAA Atmospheric Flight Mechanics Conference and Exhibit, Providence (RI), August 16-19, , No. AIAA 2004-4703, 2004. 